\title{
Single bunch longitudinal measurements at the Cornell Electron-Positron Storage Ring
}

\author{
R. Holtzapple, M. Billing, D. Hartill, and M. Stedinger \\ Laboratory of Nuclear Studies, Cornell University, Ithaca, New York 14853
}

\section{B. Podobedov}

Stanford Linear Accelerator Center, Stanford University, Stanford, California 94309

(Received 4 March 1999; published 13 March 2000)

\begin{abstract}
Measurements of the beam's bunch length in the Cornell Electron-Positron Storage Ring (CESR) have been made using a streak camera. The streak camera uses visible synchrotron radiation produced by the beam to measure its longitudinal distribution. A description of CESR, the experimental setup, the streak camera used, and systematic errors and analysis techniques of the streak camera are described in this paper. The dependence of the bunch distribution on the current and accelerating rf voltage for a single bunch CESR was measured and compared with a theoretical model of CESR. The CESR vacuum chamber impedance is determined from the measured bunch distributions and is presented in this paper.
\end{abstract}

PACS numbers: 29.27.Bd

\section{INTRODUCTION TO CESR}

In the Cornell Electron-Positron Storage Ring (CESR), electrons collide with positrons at center-of-mass energies between 9.4 and $11.2 \mathrm{GeV}$. The accelerator complex is shown in Fig. 1. The main components of the accelerator complex are (i) the electron gun which produces the electrons, (ii) the linac which accelerates the electrons to $300 \mathrm{MeV}$ to be injected into the synchrotron or is used to make positrons, (iii) the synchrotron which accelerates the electrons and positrons to their final energy where they are injected by the transfer lines into CESR, and (iv) CESR. In CESR, four five-cell rf cavities are used (for these measurements) to replace the energy lost due to synchrotron radiation. The new superconducting cavity had not been installed when the measurements were made. The energy of each beam can range between 4.7 and $5.6 \mathrm{GeV}$ but normally CESR is at either 5.289 or $5.269 \mathrm{GeV}$ depending on the requirements for high-energy physics data. The measurements were performed at both energies and the difference in bunch length for these two different energies is negligible. CESR has a $768.43 \mathrm{~m}$ circumference and the period of revolution is $2.56 \mu \mathrm{sec}$. Under normal colliding beam conditions at the time of the measurements there were nearly nine evenly spaced bunch trains with two bunches in each train for a total of 18 electron and 18 positron bunches. Vertical and horizontal electrostatic separators are used to make a pretzel orbit to ensure separation between bunch crossings at locations other than the interaction region. During electron single bunch measurements, the beam is in a pretzel orbit and for positron single bunch measurements the beam is in a flat orbit. CESR also has two wiggler magnets, located near the interaction region, which produce synchrotron radiation for the Cornell High Energy Synchrotron Source (CHESS). Opening and closing the wiggler magnets affects the longitudinal phase space of the beam, as will be discussed later.
The measurements presented in this paper were taken when only one electron or positron bunch was present in the CESR. The measurements were performed on both the electron and positron bunches when the opportunity for beam time was available. Some of the parameters of CESR are shown in Table I.

Earlier measurements of the CESR bunch length, using an X-ray sensitive photoconducting detector and the beam spectrum, as a function of current have shown no bunch

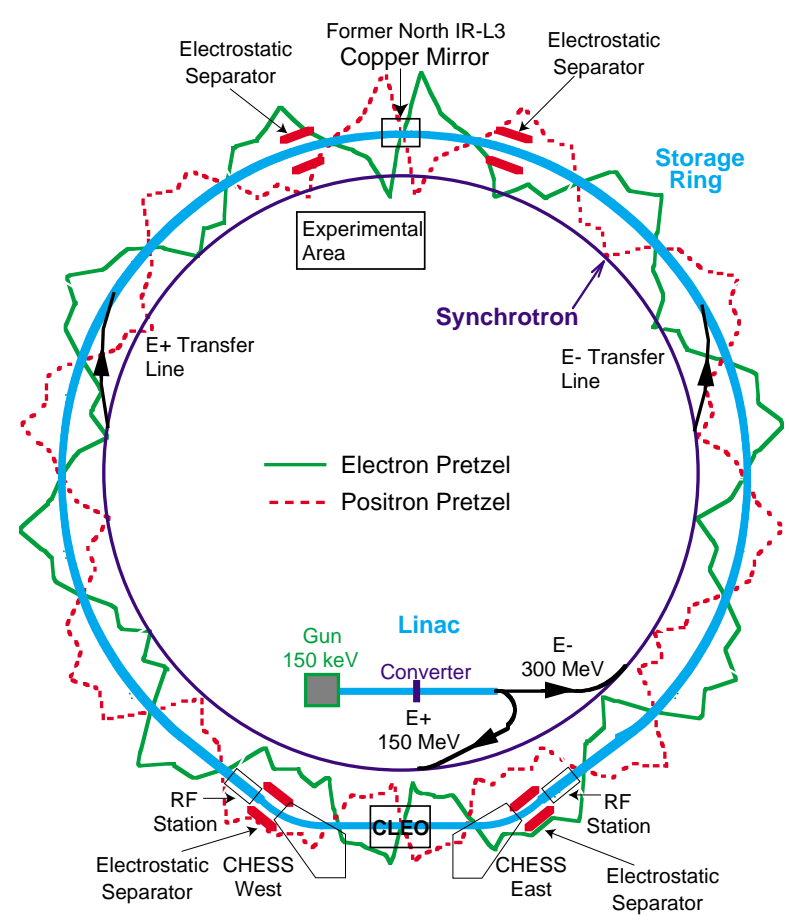

FIG. 1. (Color) The layout of the Cornell Accelerator Complex. The streak camera experimental area and the mirror used to reflect the light out of the CESR vacuum chamber are located near the former north interaction region (IR). 
TABLE I. Parameters of CESR.
Energy (on/off resonance)

Circumference

Revolution period

rf frequency

Horizontal tune $(Q x)$

Vertical tune $(Q y)$

Longitudinal tune $(Q s)$

Harmonic number

lengthening [1,2]. The resolutions of these previous measurements were not capable of measuring the effects presented in this paper.

\section{CESR SINGLE BUNCH DYNAMICS}

The longitudinal phase space in the storage ring is determined from accelerator components as well as from collective effects. In CESR, the electromagnetic fields which affect the bunch distribution are from the more than 400 magnets which guide the bunches around the accelerator, two rf accelerating stations to counteract the bunch energy loss due to synchrotron radiation, and two wiggler magnets used to create synchrotron radiation for
CHESS. The longitudinal phase space, ignoring collective effects, in a storage ring is Gaussian in both energy and length and has a phase space distribution given by [3]

$$
\Psi(\tau, \varepsilon)=\frac{1}{2 \pi \sigma_{\varepsilon} \sigma_{\tau}} \exp \left[-\frac{\varepsilon^{2}}{2 \sigma_{\varepsilon}^{2}}-\frac{\tau^{2}}{2 \sigma_{\tau}^{2}}\right],
$$

where $\varepsilon$ and $\tau$ are the energy and time deviation from a synchronous particle and $\sigma_{\tau}$ and $\sigma_{\varepsilon}$ are the standard deviation of the bunch length and energy, respectively. The standard deviation of the bunch length is given by

$$
\begin{aligned}
\sigma_{\tau} & =\left\langle\tau^{2}\right\rangle^{1 / 2}=T_{0} \sqrt{\frac{\alpha E_{0}}{2 \pi h e V_{\mathrm{rf}} \sin \phi_{s}}}\left(\frac{\sigma_{\varepsilon}}{E_{0}}\right) \\
& =\frac{\alpha}{\Omega_{s}} \sqrt{C_{q} E_{0}^{2}\left(\frac{I_{3}}{2 I_{2}+I_{4}}\right)},
\end{aligned}
$$

where $I_{2}, I_{3}$, and $I_{4}$ are the synchrotron integrals. The term $\alpha$ is the momentum compaction, $\omega_{\text {rev }}$ and $\omega_{\text {rf }}$ are the revolution and $\mathrm{rf}$ frequencies, respectively, $E_{0}$ is the nominal energy, $h=\omega_{\mathrm{rf}} / \omega_{\text {rev }}$ is the harmonic number, $\phi_{s}=\cos ^{-1}\left(U_{0} / e V_{\mathrm{rf}}\right)$ is the synchronous phase, $V_{\mathrm{rf}}$ is the rf accelerating voltage, and $U_{0}$ is the energy loss per turn for a synchronous particle. The synchrotron integrals, $I_{1}-I_{4}$, in their integral and summation forms are given by

$$
\begin{gathered}
I_{1}=\oint\left(\frac{\eta}{\rho(s)}\right) d s=\sum_{i} \frac{l_{i}}{\rho_{i}}\langle\eta\rangle_{i}, \quad I_{2}=\oint\left(\frac{1}{\rho^{2}(s)}\right) d s=\sum_{i} \frac{l_{i}}{\rho_{i}^{2}} \\
I_{3}=\oint\left|\frac{1}{\rho(s)}\right|^{3} d s=\sum_{i} \frac{l_{i}}{\left|\rho_{i}\right|^{3}}, \quad I_{4}=\oint \frac{[1+2 k(s)] \eta}{\rho^{3}(s)} d s=\sum_{i}\left[\frac{l_{i}}{\rho_{i}^{3}}\langle\eta\rangle_{i}+2 l_{i}\left\langle\frac{K \eta}{\rho^{3}}\right\rangle_{i}\right],
\end{gathered}
$$

where $1 / \rho(s)=e c B(s) / E_{0}$ is the inverse of the radius of curvature for a dipole magnet, $\eta(s)$ is the dispersion, and $K(s)=\left(e c / E_{0}\right)(\partial B / \partial x)$ is proportional to the gradient of the field. The energy lost per turn, $U_{0}$, is also derived from the synchrotron integrals and is given by

$$
U_{0}=\frac{2}{3} r_{e} \frac{E_{0}^{4}}{\left(m c^{2}\right)^{3}} I_{2}
$$

The synchrotron integrals can be determined for the CESR ring by knowing the length and radius of curvature of the dipole magnets (Table II), the dispersion, gradient of the fields, and wiggler magnet parameters. The synchrotron integrals that reflect CESR when the streak camera experiments were performed are denoted in Table III.

The energy spread of CESR is dependent on the energy and bending radius. The energy fluctuations depend on the bending radius and do not change as the bunch distribution changes, so the bunch remains Gaussian in energy. The energy spread can be determined from the synchrotron integrals to give

$$
\left(\frac{\sigma_{\varepsilon}}{E_{0}}\right)=\sqrt{\frac{C_{q} \gamma^{2} I_{3}}{2 I_{2}+I_{4}}}
$$

where the constant $C_{q}=3.84 \times 10^{-13} \mathrm{~m}$. Measuring the energy spread in a storage ring to a high degree of accuracy is a difficult task. Ideally, making measurements with a high-resolution camera or wire scanner located in a high dispersion region is the preferred method to measure the energy spread. Presently the only energy-spread measurement on CESR was done by the CLEO detector on the $1 s$ and $2 s$ resonance. The result of these measurements is that the energy spread on the $1 s$ and $2 s$ resonance is consistent with being constant as a function of current.

TABLE II. The magnet length and radius of curvature for the 84 dipole magnets in CESR.

\begin{tabular}{ccc}
\hline \hline & $\begin{array}{c}\text { Magnet length } \\
\text { Number of magnets }\end{array}$ & $\begin{array}{c}\text { Radius of curvature } \\
\rho(\mathrm{m})\end{array}$ \\
\hline 4 & 6.572 & 58.603 \\
62 & 6.573 & 87.891 \\
4 & 2.945 & 140.626 \\
8 & 3.236 & 31.654 \\
2 & 1.644 & 87.892 \\
2 & 3.287 & 87.892 \\
2 & 3.176 & 34.818 \\
\hline \hline
\end{tabular}


TABLE III. The synchrotron integral for CESR for the case when the wiggler magnets are open (CHESS is not collecting data) or closed.

\begin{tabular}{ccc}
\hline \hline & Wigglers open & Wigglers closed \\
\hline$I_{1}$ & $8.791 \mathrm{~m}$ & $8.791 \mathrm{~m}$ \\
$I_{2}$ & $9.336 \times 10^{-2} \mathrm{~m}^{-1}$ & $1.047 \times 10^{-1} \mathrm{~m}^{-1}$ \\
$I_{3}$ & $1.716 \times 10^{-3} \mathrm{~m}^{-2}$ & $2.372 \times 10^{-3} \mathrm{~m}^{-2}$ \\
$I_{4}$ & $2.088 \times 10^{-3} \mathrm{~m}^{-1}$ & $2.744 \times 10^{-3} \mathrm{~m}^{-1}$ \\
$I_{5}$ & $3.890 \times 10^{-4} \mathrm{~m}^{-1}$ & $5.386 \times 10^{-4} \mathrm{~m}^{-1}$ \\
$U_{0}$ & $1.0290 \mathrm{MeV}$ & $1.1541 \mathrm{MeV}^{-4}$ \\
$\sigma_{\varepsilon} / E_{0}$ & $6.115 \times 10^{-4}$ & $6.782 \times 10^{-4}$ \\
$\sigma_{Z}=c \sigma_{\tau}$ & $1.565 \times 10^{-2} \mathrm{~m}$ & $1.739 \times 10^{-2} \mathrm{~m}$ \\
\hline \hline
\end{tabular}

It should be noted that the energy spread and bunch length from Eqs. (2) and (3) are their equilibrium values at low currents. The relationship between $\sigma_{\tau}$ and $\sigma_{\varepsilon} / E_{0}$ depends on the local slope of the rf waveform and beam induced fields can distort the bunch shape even while the energy spread remains Gaussian. As the intensity of the bunch increases, collective effects that modify the slope of the rf wave have to be included. In CESR there are several phenomena that affect the single bunch distribution.

\section{A. Beam induced voltage-potential well distortion}

For single particle motion, the bunch distribution in equilibrium has a Gaussian distribution and the length depends upon the rf accelerating voltage. In the same manner that the rf accelerating fields affect the bunch distribution, beam induced voltages from longitudinal wakefields also influence the shape of the bunch distribution. The beam induced voltages are categorized into three different types and their influence on the longitudinal dynamics of CESR will be examined.

With the assumption that the energy spread remains constant, the beam induced voltages described below result in potential well distortion of the bunch distribution. With potential well distortion, the bunch distribution is static but distorted from a Gaussian distribution by the beam induced voltage [4]. We observe in CESR a greater distortion for higher beam intensity. There is another possibility, which is that the distribution is not static but instead performs a collective oscillation. This is discussed later.
The steady state distribution can be determined by solving the Vlasov equation where $\Psi(\varepsilon, \tau)$ is the phase space distribution normalized to unity $[5,6]$. The Vlasov equation is used to describe a multiparticle system that is influenced by electromagnetic fields. In our case, the Vlasov equation is

where

$$
\frac{\partial \Psi}{\partial t}+\dot{\tau} \frac{\partial \Psi}{\partial \tau}+\dot{\varepsilon} \frac{\partial \Psi}{\partial \varepsilon}=0
$$

$$
\dot{\tau}=-\alpha \frac{\varepsilon}{E_{0}}
$$

and

$$
\dot{\varepsilon}=e \frac{V(\tau)-U(\varepsilon)}{T_{0}} .
$$

The longitudinal phase space can be modified to include the beam induced voltage terms. Now the term $V(\tau)$ includes the rf accelerating voltage and the beam induced voltage. The induced voltage can be expressed in terms of three different categories: one which has time dependence identical to the input current (resistive part), the second which has time dependence like the derivative of the input current (inductive part), and the third which has time dependence like the integral of the input current (capacitive part). Including the above terms, the function $V(\tau)$ can be written as [5]

$$
\begin{aligned}
V(\tau)= & V_{\mathrm{rf}}(\tau)+V_{b}(\tau) \\
= & V_{\mathrm{rf}} \cos (\omega \tau+\varphi)+R I_{b}(\tau) \\
& +L \frac{d I_{b}(\tau)}{d \tau}+\frac{1}{C} \int_{-\infty}^{\tau} d \tau^{\prime} I_{b}\left(\tau^{\prime}\right),
\end{aligned}
$$

where $V_{\mathrm{rf}}(\tau)$ is the rf accelerating voltage and the three other terms are the induced voltages from the resistive $(R)$, capacitive $(C)$, and inductive $(L)$ impedance of the CESR vacuum chamber. Also,

$$
I_{b}(\tau)=Q \int_{-\infty}^{\infty} \Psi(\varepsilon, \tau) d \varepsilon=Q \psi(\tau),
$$

where $Q$ is the total charge in the bunch. The bunch shape is a function of the resistance, capacitance, and inductance.

Under the assumption that the energy distribution remains unchanged, the steady state longitudinal distribution of the bunch may be written as [5]

$$
\frac{\partial \psi(\tau)}{\partial \tau}=\frac{-e E_{0} \psi(\tau)}{\sigma_{\varepsilon}^{2} \alpha T_{0}}\left[\frac{V_{\mathrm{rf}} \cos (\omega \tau+\varphi)+Q R \psi(\tau)+\frac{Q}{C} \int_{-\infty}^{\tau} d \tau^{\prime} \psi\left(\tau^{\prime}\right)-U_{0}}{1+\frac{e E_{0} Q L \psi(\tau)}{\sigma_{\varepsilon}^{2} \alpha T_{0}}}\right]
$$

The longitudinal distribution $\psi(\tau)$ can be determined numerically by integrating Eq. (4). The general formalism for calculating the distribution of particles in CESR using Vlasov's theory is described elsewhere [5]. The simulations presented here are an updated version of the soft- ware developed earlier that numerically integrates Eq. (4) to determine the bunch distribution for the given input parameters. The following are three examples of the bunch distribution determined by numerically integrating Eq. (4) with the CESR parameters listed in Table IV. 
TABLE IV. The parameters used in the numerical analysis to determine the CESR bunch distribution. The parameters listed here are representative of the CESR beam when the wiggler magnets are closed.

\begin{tabular}{cc}
\hline \hline$E_{0}$ & $5.289 \mathrm{GeV}$ \\
$\sigma_{\varepsilon} / E_{0}$ & $6.782 \times 10^{-4}$ \\
$T_{0}$ & $2.56 \times 10^{-6} \mathrm{sec}$ \\
$\alpha$ & $1.14 \times 10^{-2}$ \\
$V_{\mathrm{rf}}$ & $6.87 \mathrm{MV}$ \\
$U_{0}$ & $1.15 \mathrm{MeV}$ \\
\hline \hline
\end{tabular}

\section{Resistive impedance beam induced voltage}

When the beam induced voltage has a time dependence which approximately matches that of the charge distribution, it is considered a resistive beam induced voltage; this will result in the bunch distribution becoming asymmetric in shape. The vacuum elements in CESR that give rise to resistive beam induced voltage are the rf and other large cavities. A pure resistive beam induced voltage is proportional to the current density and is given by

$$
V_{b}(\tau)=R I_{b}(\tau),
$$

where $R$ is the resistance and $I_{b}$ is the bunch current. The longitudinal distribution for a resistive beam induced voltage can be numerically integrated (ignoring the capacitive and inductive terms) using Eq. (4). Figure 2 is an example of the CESR bunch distribution at a fixed current for several different resistance values. As the resistance is increased the bunch shifts in synchronous phase to compensate for the increased higher order mode loss which causes the bunch distribution to tilt, becoming more asymmetric in shape.

The simulated bunch distributions are fit to an asymmetric Gaussian function (described later in this paper) in

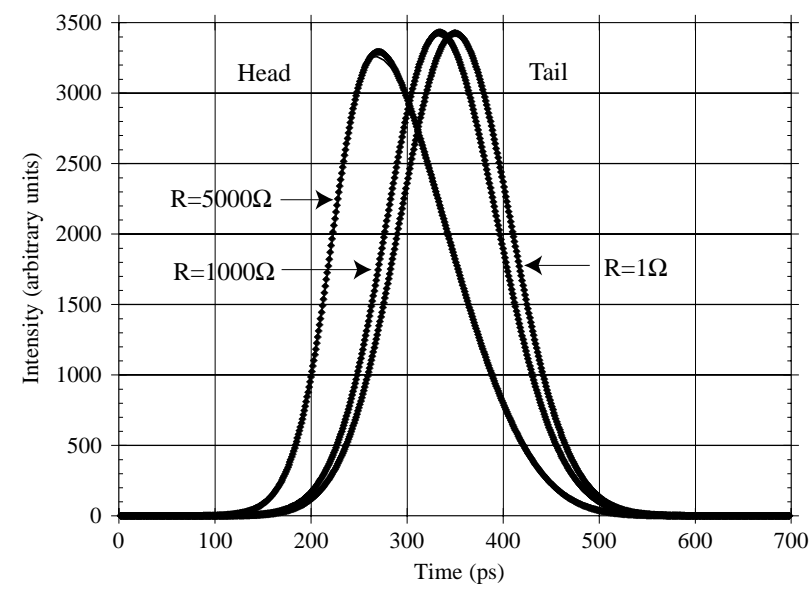

FIG. 2. A simulation of the CESR bunch distribution fit to an asymmetric Gaussian function at several different resistance values. The single bunch current for this simulation is $20 \mathrm{~mA}$ and the CESR machine parameters used to determine the bunch distribution are listed in Table IV. order to quantify the change in the length and asymmetry of the distribution. The bunch length and asymmetry factor as a function of current and resistance are plotted in Figs. 3(a) and 3(b). The bunch length does increase as a function of current for a fixed resistance.

The resistive impedance of the CESR vacuum chamber has been measured by two different methods: (i) measuring the higher order mode loss and (ii) measuring the bunch distribution. The higher order mode loss factor, $k_{\mathrm{HOM}}$, was measured on CESR to be [7]

$$
\begin{aligned}
k_{\mathrm{HOM}, \text { open }} & =7.53 \pm 1.13 \frac{\mathrm{V}}{\mathrm{pC}} \\
k_{\mathrm{HOM}, \text { closed }} & =6.64 \pm 1.00 \frac{\mathrm{V}}{\mathrm{pC}},
\end{aligned}
$$
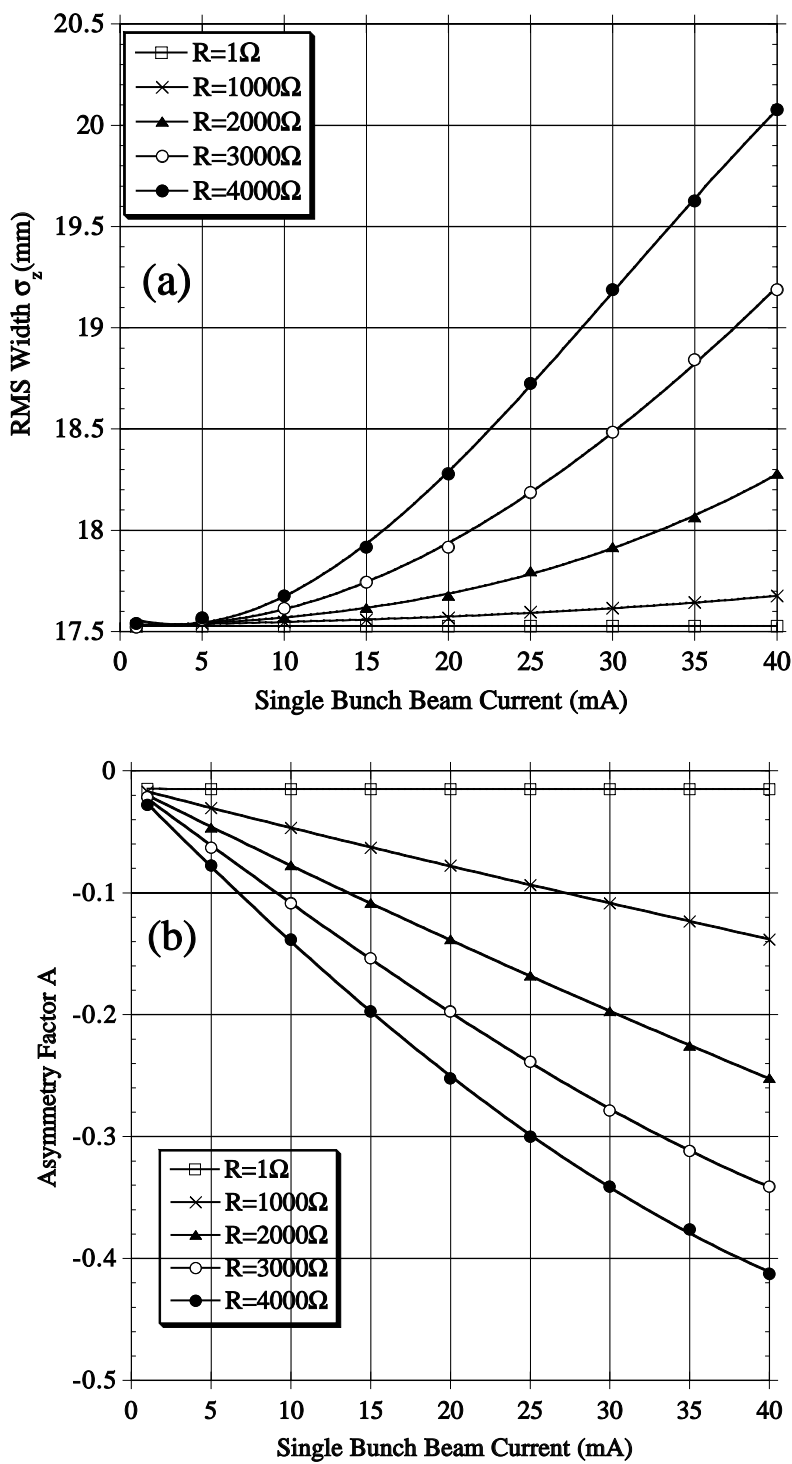

FIG. 3. Results of the simulation of the CESR (a) bunch length and (b) asymmetry factor as a function of current at several different resistance values. 
with the wiggler magnets open and closed, respectively. For a Gaussian bunch shape, the resulting resistive impedance is

$$
\begin{gathered}
R_{\text {open }}=1393 \pm 209 \Omega \\
R_{\text {closed }}=1365 \pm 205 \Omega,
\end{gathered}
$$

with the wiggler magnets open and closed, respectively. Using the bunch distribution measurements by the streak camera, the resistive impedance can also be determined and is presented in the analysis section of this paper.

\section{Inductive impedance beam induced voltage}

When the slope of the beam induced voltage is opposite to the slope of the rf accelerating voltage it is considered to be inductive and, as a result, bunch lengthening occurs. If the impedance is modeled as pure inductance, the beam induced voltage is proportional to the derivative of the charge density and is expressed as

$$
V_{b}(\tau)=L \frac{d I_{b}}{d \tau},
$$

where $L$ is the inductance. The vacuum components in storage rings that cause inductive voltages are masks, bellows, and abrupt small (with respect to the bunch length) transitions in vacuum chamber. Figure 4 is an example of numerically integrating Eq. (4) for the CESR bunch distribution at a fixed current for several different values of the inductance. Notice that as the inductance is increased the peak of the distribution becomes flatter and it remains symmetric around the origin. This symmetry is a result of the bunch having no net energy loss from a perfect inductive impedance.

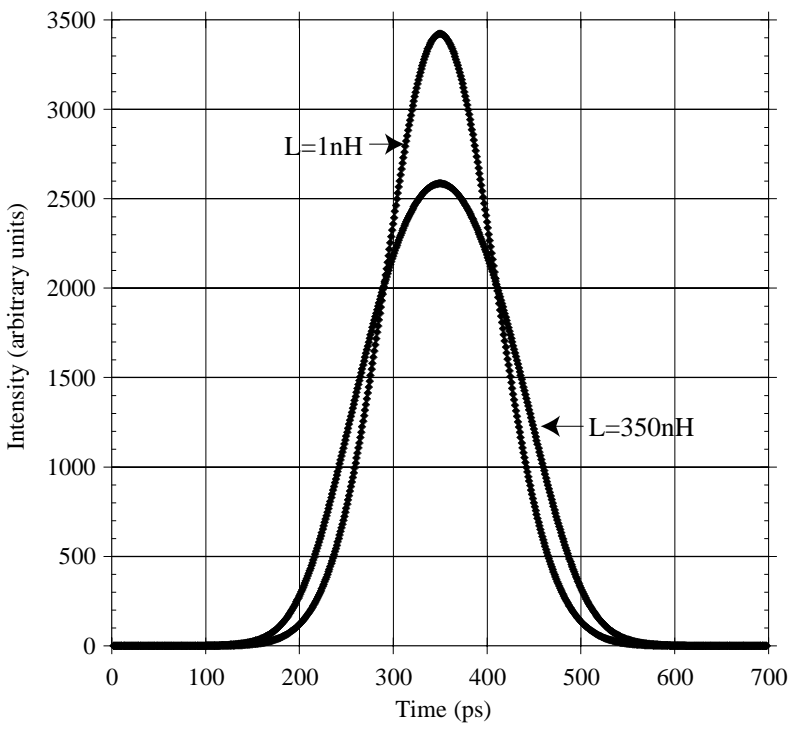

FIG. 4. A simulation of the CESR bunch distribution at two different inductance values. The parameters used to determine the bunch distribution are listed in Table IV, and the bunch current is $20 \mathrm{~mA}$. The bunch distributions are not fit to a function but instead the full width at half maximum of the bunch is used to characterize the bunch length.

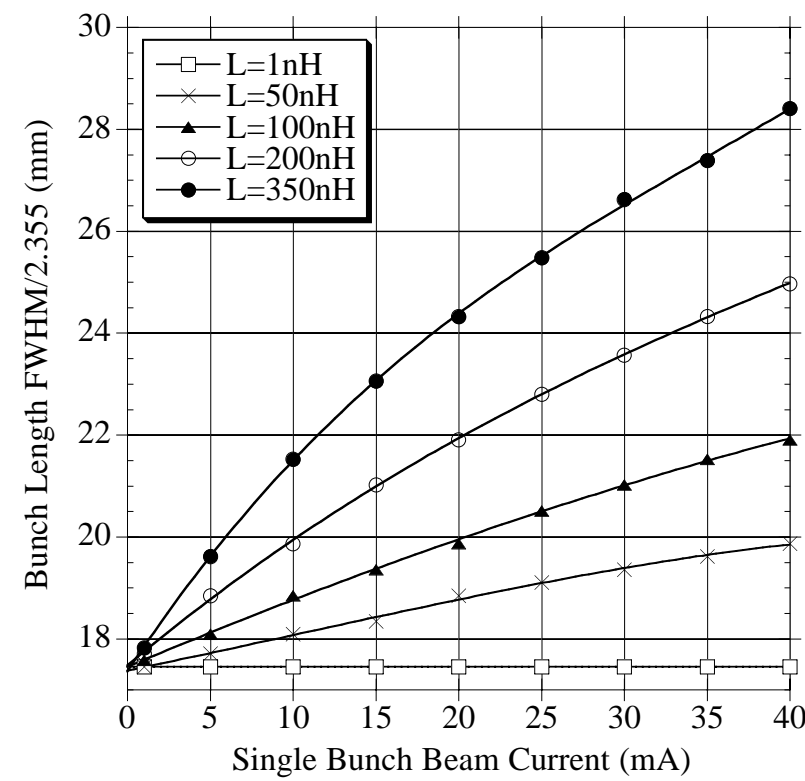

FIG. 5. The results of the simulation of the CESR bunch length as a function of current at several different inductance values.

As the inductance increases the bunch shape deviates from a Gaussian distribution. The bunch length is determined by calculating the full width at half maximum (FWHM) divided by $2 \sqrt{2 \ln 2}$ (2.355). Figure 5 is the simulated bunch length as a function of current for several different inductance values. The bunch length increases as a function of current for a fixed inductance.

\section{Capacitive impedance beam induced voltage}

When the beam induced voltage is proportional to the integral of the current it is considered to be capacitive and, as a result, bunch shortening occurs. If the beam induced voltage is modeled as pure capacitive, the beam induced voltage is proportional to the integral of the current density and is expressed as

$$
V_{b}(\tau)=\frac{1}{C} \int_{-\infty}^{\tau} d \tau^{\prime} I_{b}\left(\tau^{\prime}\right),
$$

where $C$ is the capacitance. The vacuum chamber components in a storage ring that cause capacitive induced voltages are $\mathrm{rf}$ cavities and other large (with respect to the bunch length) discontinuities in the vacuum chamber. Figure 6 is an example of the CESR bunch distribution, numerically integrated from Eq. (4), at a fixed current for several different capacitive values. As the inverse of the capacitance is increased, the bunch is shifted in synchronous phase to compensate for energy used in creating the capacitive voltage and, as a result, the bunch shortens.

The bunch length for the simulated bunch distribution as a function of current and capacitance is determined by calculating the full width at half maximum divided by 2.355 , as plotted in Fig. 7 . The bunch length does decrease as a function of current for a fixed capacitance. 


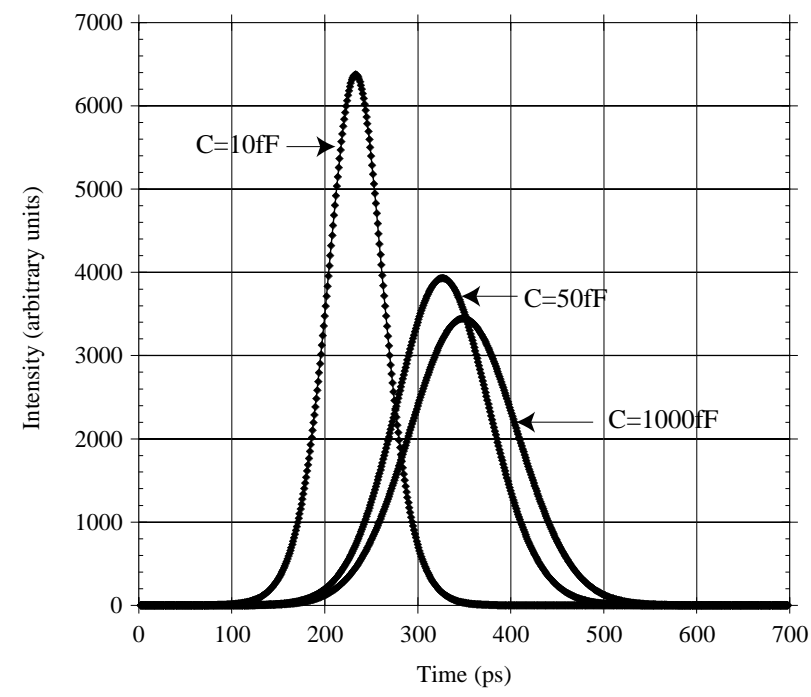

FIG. 6. A simulation of the CESR bunch distribution at several different values of the capacitance. The parameters used to determine the bunch distribution are listed in Table IV, and the current of the bunch for the simulations is $20 \mathrm{~mA}$.

The above examples illustrate how the beam induced voltage can change the bunch distribution in CESR. In reality, the impedance of the CESR vacuum chamber consists of resistive, inductive, and capacitive parts. For the bunch lengths measured in CESR, the reactive impedance is dominated by the inductance so the capacitive impedance can be ignored. In CESR, the bunch shape is fairly Gaussian at low current and becomes asymmetric, due to the resistive impedance, as the current is increased. In principle, the particle distributions measured in CESR can be used to measure the dominant components of the impedance that will be presented later.

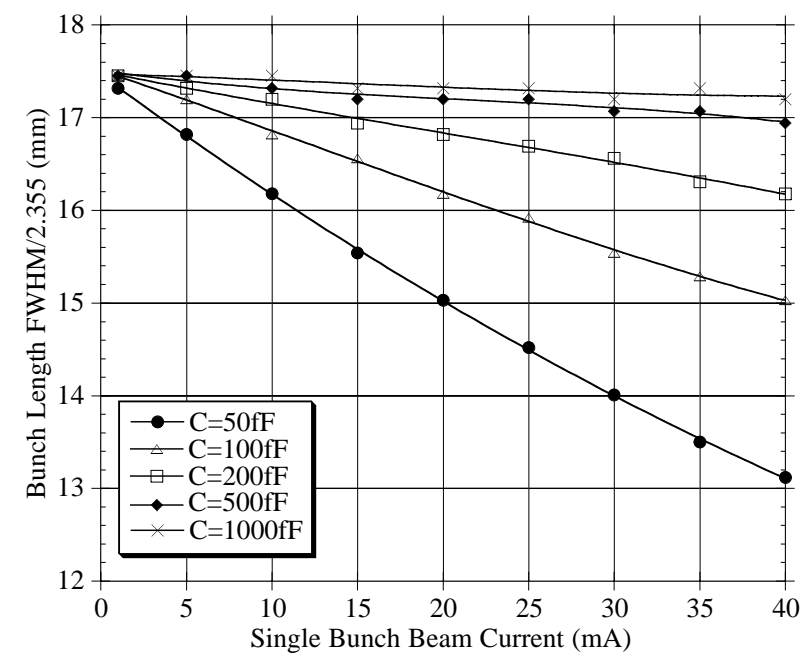

FIG. 7. The results of the simulation of the CESR bunch length as a function of current at several different capacitive values. The bunch length is the FWHM of the distribution divided by 2.355 .

\section{B. Longitudinal instabilities}

The discussion above has assumed that the longitudinal distribution of the beam is time independent. This cannot be taken for granted when longitudinal wakefields are present. What usually happens is that the beam becomes unstable above some threshold current. The general methods of analysis of instabilities are to linearize Vlasov's equation for small perturbations or to use computer simulations. One result is that the energy spread no longer remains constant as it does with potential well distortion. The instability causes energy and bunch shape oscillations to occur and these are visible as synchrotron sidebands of the bunch's rotation harmonics and as an increase in the energy spread of the bunch. Due to our inability to measure the energy spread, the beam spectrum measurements suffice to indicate an instability onset.

There is a longitudinal instability in CESR, but it is a multibunch effect and is not seen with a single bunch. Potential well distortion from beam induced voltage is the dominant effect with single bunch operation.

\section{EXPERIMENTAL SETUP}

The streak camera uses synchrotron radiation produced by the accelerator dipole magnets to determine the longitudinal bunch distribution. When the bunch travels through the dipole magnet it emits a pulse of synchrotron light which contains the longitudinal distribution of the bunch. The electrons (or positrons) in the head of the bunch emit light before the electrons in the tail of the bunch, and the longitudinal intensity of this pulse of light is proportional to the number of particles located longitudinally in the bunch. The light pulse has the longitudinal profile encoded in its length and the streak camera will decode this information. The synchrotron light pulse is transported from the source out of the vacuum chamber to a safe location shielded from radiation where the streak camera measurements can be made. The location in CESR where the measurements were performed is ideal because synchrotron light from both electrons and positrons can be observed from identical magnets for both beams, and the same optics can be used to deliver the light to the streak camera. The dipole magnet and optics used to transport the light out of the CESR vacuum chamber is shown in Fig. 8.

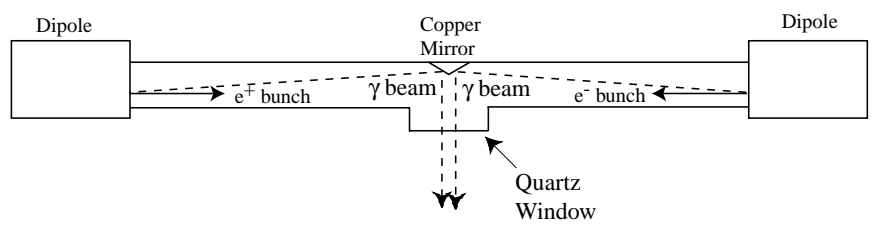

FIG. 8. A cross sectional view looking vertically down on the vacuum chamber where the copper mirror is located in CESR. Synchrotron light from both beams is emitted in the dipole magnet and strikes the mirror that transports the light out of the vacuum chamber. 
TABLE V. The dipole magnet and synchrotron radiation parameters for the streak camera experiment. All the parameters are for an energy in CESR of $5.289 \mathrm{GeV}$.

\begin{tabular}{lc}
\hline \hline Length & $2.9453 \mathrm{~m}$ \\
Pole tip field & $1.25 \mathrm{kG}$ \\
$\int B \cdot d l$ & $3.70 \mathrm{kG} \mathrm{m}$ \\
Bend angle & $20.944 \mathrm{mrad}$ \\
Radius of curvature & $140.628 \mathrm{~m}$ \\
Synchrotron radiation opening angle & $0.966 \mathrm{mrad}$ \\
Synchrotron radiation critical energy & $2.33 \mathrm{KeV}$ \\
Synchrotron radiation critical wavelength & $0.511 \mathrm{~nm}$ \\
\hline \hline
\end{tabular}

The synchrotron light is emitted by the beam traveling through a dipole magnet adjacent to the straight section in the old detector location called L3 (Fig. 1). The synchrotron light is reflected by a highly polished water cooled copper mirror which is cut at a $45^{\circ}$ angle to the vacuum chamber wall, and the visible light passes through a quartz window out of the vacuum chamber. The copper mirror was polished by hand with a jig that holds the mirror at an angle of $45^{\circ}$ to a flat polishing wheel. A diamond polishing compound as fine as $1 \mu \mathrm{m}$ was used to ensure the flatness and reflectivity of the mirror. The mirror was designed to protrude into the vacuum chamber wall $1 \mathrm{~cm}$. After the mirror, the synchrotron light is then transported over the synchrotron and through a hole in the steel of the old detector. The light is transported by a series of seven mirrors, which includes two periscopes, over a distance of approximately $56 \mathrm{ft}$ from the light source to the location of the streak camera. The second mirror in the setup can be moved transversely by remote control to allow for tuning of either the electron or positron synchrotron light. There are three lenses used to focus the light on the streak camera slit. The first lens has a focal length of $1.5 \mathrm{~m}$ and is located just inside the radiation shielded experimental hall. The second lens is a cylindrical one, which spreads the light horizontally across the slit of the camera. The third lens is located approximately 3 in. from the camera and is used for additional focusing on the slit of the camera. The properties of the dipole magnets which produce the synchrotron light is shown in Table V.

\section{DESCRIPTION OF THE STREAK CAMERA}

The streak camera is a device to measure ultrafast pulsed light intensity versus time. A detailed explanation of the operation of a streak camera can be found elsewhere [8]. The streak camera used in this experiment was a Hamamatsu model N3373-02 camera made by Hamamatsu Photonics. The basic components of the streak camera are shown in Fig. 9, and they are the variable input slit, input optics, photo cathode, accelerating mesh, deflection plate, microchannel plate, phosphor screen, output optics, charge-coupled device camera, and analog-to-digital readout system. The streak camera resolution is dependent on the streak speed of the cam-

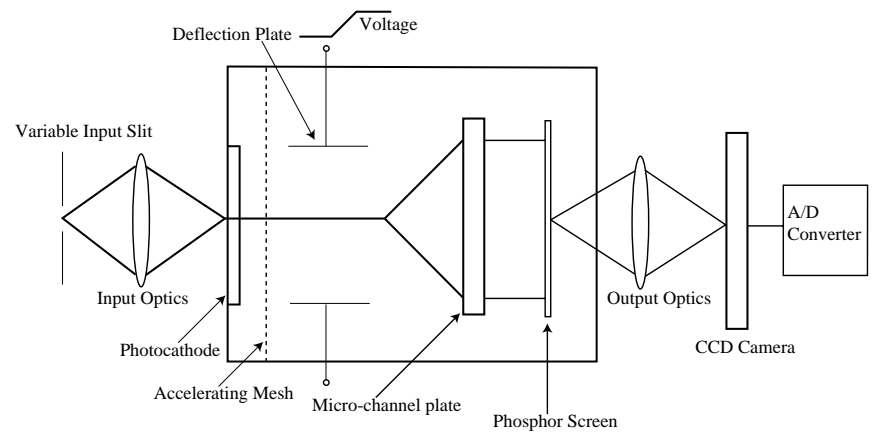

FIG. 9. The layout of the Hamamatsu streak camera.

era and on the slit width used. This particular streak camera has four different streak speeds: $60 \mathrm{ps} / 10 \mathrm{~mm}$, $200 \mathrm{ps} / 10 \mathrm{~mm}, 500 \mathrm{ps} / 10 \mathrm{~mm}$, and $1.2 \mathrm{~ns} / 10 \mathrm{~mm}$.

\section{METHOD OF DATA ANALYSIS}

The streak camera takes images of the bunch distribution, which are $512 \times 512$ pixels in size. A selected area can be chosen such that the vertical columns in that area are projected on the horizontal axis. This creates a profile that can be fit to a function that characterizes the distribution of the bunch.

The bunch distribution in CESR deviates from a pure Gaussian distribution due to potential well distortion. The function used to characterize the bunch shape is an asymmetric Gaussian function. The longitudinal profiles of the beam distribution are fitted to an asymmetric Gaussian function with a constant background given by

$$
I(z)=I_{0}+I_{1} \exp \left\{-\frac{1}{2}\left(\frac{(z-\bar{z})}{[1+\operatorname{sgn}(z-\bar{z}) A] \sigma}\right)^{2}\right\},
$$

where $I_{0}=$ pedestal and $I_{1}=$ peak of the asymmetric Gaussian. The term $\operatorname{sgn}(z-\bar{z}) A$ is the asymmetry factor that parameterized the shape of the asymmetric Gaussian. Figure 10 is a profile of the selected area with a fit to an asymmetric Gaussian function. The longitudinal profile of the beam distribution in CESR is $\chi^{2}$ minimized using the minimization package Minuit [9]. A $\chi^{2}$ minimization was performed on each streak camera picture

$$
\chi^{2}=\sum_{i}^{n} \frac{\left[I\left(z_{i} ; A, I_{0}, I_{1}, \bar{z}, \sigma\right)-x_{i}\right]^{2}}{x_{i}},
$$

where $x_{i}$ is the digitized signal from the streak camera profile. The fit returns the mean $\bar{z}$, asymmetry factor $A$, background level $I_{0}$, peak of the asymmetric Gaussian $I_{1}$, and width $\sigma$ of the distribution. The pertinent information retrieved from the asymmetric Gaussian function is the rms width

$$
\begin{aligned}
\sigma_{z} & =c \sigma_{\tau}=\text { rms width } \\
& =\left\langle(z-\langle z\rangle)^{2}\right\rangle^{1 / 2}=\left[1+\left(3-\frac{8}{\pi}\right) A^{2}\right]^{1 / 2} \sigma,
\end{aligned}
$$

the mean of the distribution 


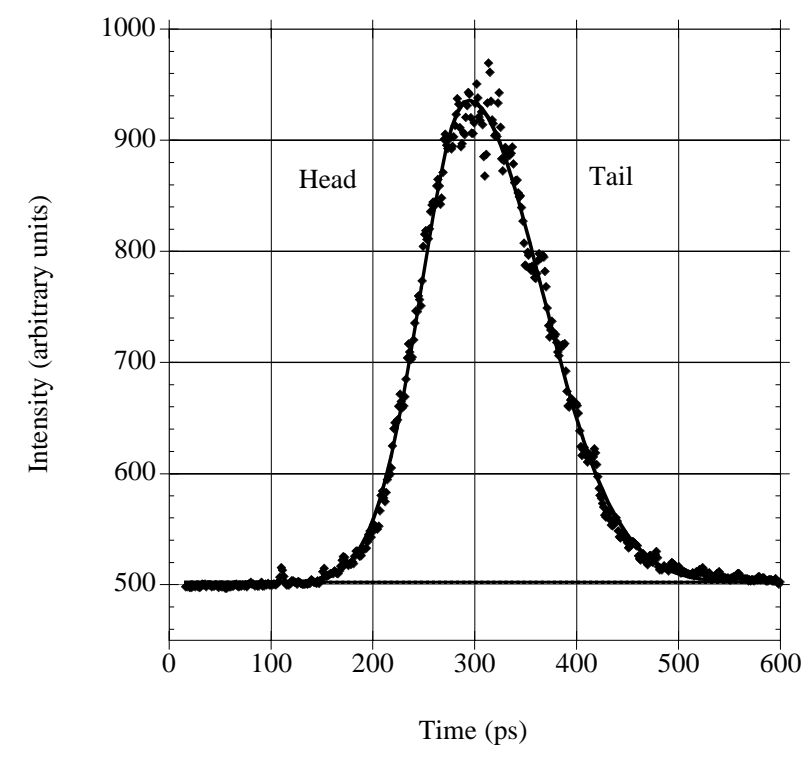

FIG. 10. A streak camera profile of the longitudinal distribution of the CESR beam fit to an asymmetric Gaussian function. The head of the bunch (early in time) is to the left. This particular profile was taken with a single bunch current of $30.7 \mathrm{~mA}$.

$$
\langle z\rangle=\text { mean }=\bar{z}+2 \sqrt{\frac{2}{\pi}} A \sigma,
$$

and the asymmetry factor $A$. These quantities are the quoted results from the measurements.

Many experiments were performed on CESR with different machine parameters. During each experiment a set of data, which consists of at least ten streak camera pictures, was taken under the same accelerator conditions. The mean rms width $\sigma_{z}$ and asymmetry factor $A$ from each experiment were used to examine the pulse to pulse fluctuations in the beam distribution. Fitting each streak camera distribution to the mean rms width and asymmetry factor and letting the area and mean position of the asymmetric Gaussian function vary, the difference, or residual, between the fit and the data shows variations between an average distribution and individual pictures. The residual, $R_{i j}$, is the $j$ th pixel from the experiment with $i$ streak camera profiles. The residual was determined from the expression

$$
R_{i j}=x_{j}-I\left(z_{j} ; \bar{A}, I_{0}, I_{1}, \bar{z}, \bar{\sigma}\right),
$$

where $I\left(z_{j} ; \bar{A}, I_{0}, I_{1}, \bar{z}, \bar{\sigma}\right)$ is determined by doing a $\chi^{2}$ fit to the asymmetric Gaussian function using the mean sigma $(\bar{\sigma})$ and mean asymmetry factor $(\bar{A})$ from the given experiment (these quantities are held constant in the fit), and $x_{j}$ is the data from the $j$ th pixel. The mean $(\bar{z})$, pedestal $I_{0}$, and peak $\left(I_{1}\right)$ of the expression $I\left(z_{j} ; \bar{A}, I_{0}, I_{1}, \bar{z}, \bar{\sigma}\right)$ are the fit variables. Figure $11(\mathrm{a})$ is an example of the bunch distribution in CESR that was fit to the asymmetric Gaussian function with the mean sigma and asymmetry factor, and 11(b), the residual from this fit, is determined from Eq. (5).

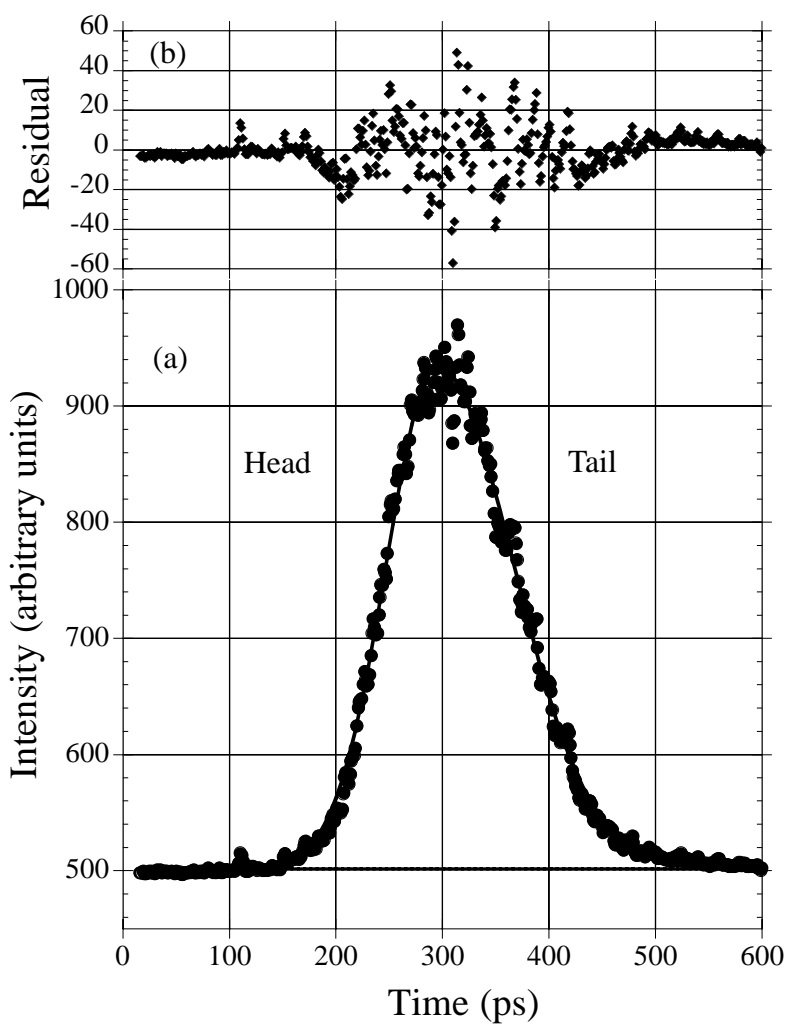

FIG. 11. (a) A single streak camera picture of the CESR bunch distribution when fit to an asymmetric Gaussian function with the mean rms width and asymmetry factor from the experimental data set. This profile was measured with a single bunch of $30.7 \mathrm{~mA}$. (b) The residual is the difference between the data and the fit.

The residuals from each experiment can also be summed to give the mean residual. The mean residual from an experimental data set is determined by summing up all the individual residuals in the data set. Because each profile has a different mean position, the residual from each profile is shifted so the mean of the asymmetric Gaussian is at the origin. The residual pixel intensity from each profile is then summed and averaged in $2 \mathrm{ps}$ bins to remove the granularity of the calibration curve. The mean residual for the $j$ th 2 ps bin is given by

$$
\bar{R}_{j}=\frac{\sum_{i=1}^{m}\left(x_{j}-I\left(z_{j} ; \bar{A}, I_{0}, I_{1}, \bar{z}, \bar{\sigma}\right)\right)}{m},
$$

where $I\left(z_{j} ; \bar{A}, I_{0}, I_{1}, \bar{z}, \bar{\sigma}\right)$ is the asymmetric Gaussian fit to the data when the mean rms width and asymmetry value are held constant and $m$ is the number of pictures taken during the experiment. Figure 12 is an example of the average residual for the experiment when the current was $30.7 \mathrm{~mA}$.

It should be noted that caution should be taken when dealing with residuals because an instability oscillates and, if the camera's data taking is not correlated with this oscillation, the residual will wash out over many pictures. 


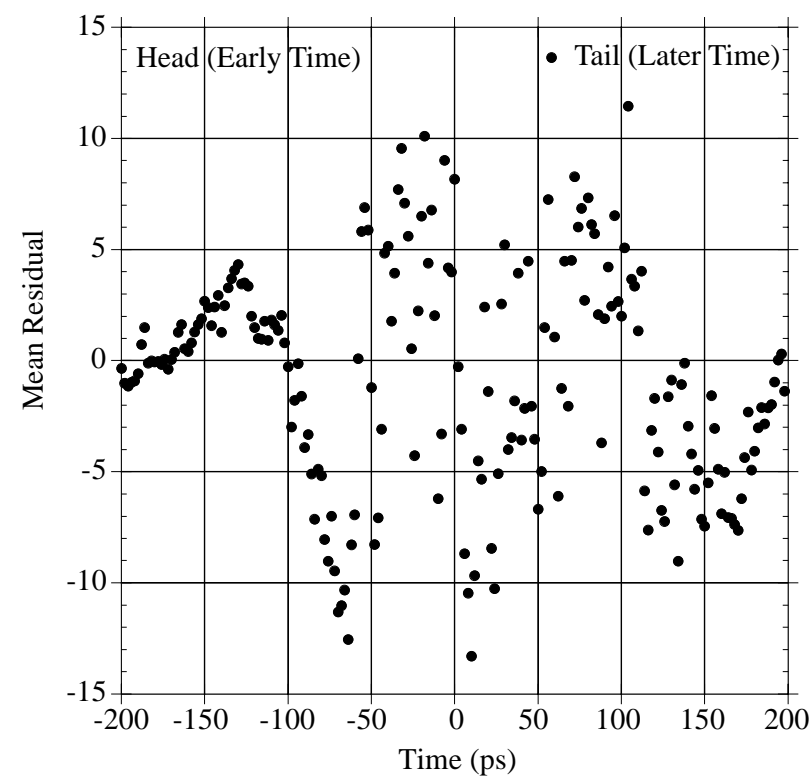

FIG. 12. The mean residual, which is a sum of the 15 residuals, summed when the mean of the distribution is shifted to the origin. The single bunch current during this measurement was $30.7 \mathrm{~mA}$.

\section{STREAK CAMERA OPERATIONS}

\section{A. Time calibration}

The streak camera tube was calibrated at the Hamamatsu Corporation and the time calibration curve for the streak speed used in these measurements is shown in Fig. 13. The calibration data was fit to a polynomial curve that was used in the analysis of the data. Each pro-

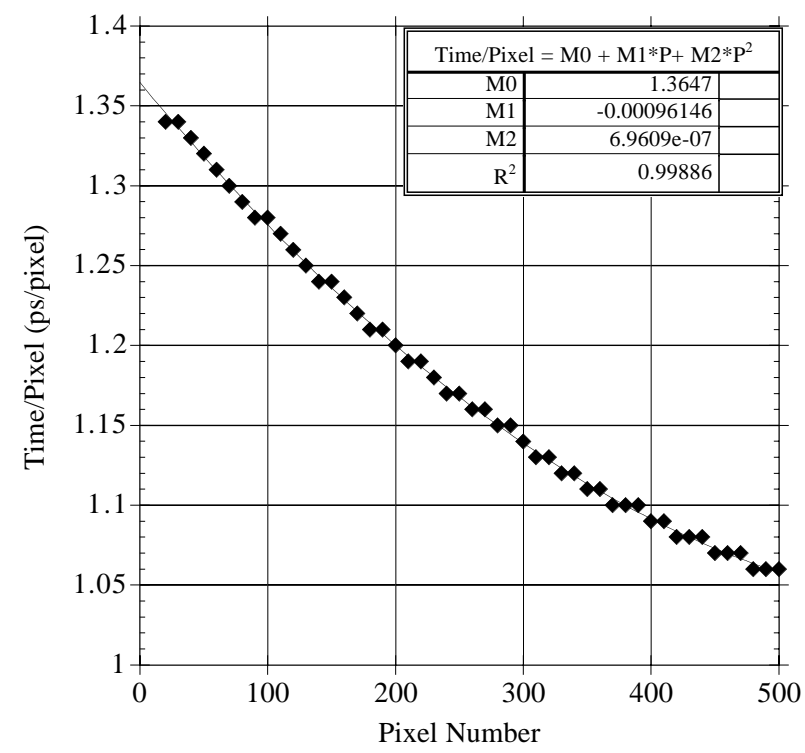

FIG. 13. The linearity curve for the $500 \mathrm{ps} / 10 \mathrm{~mm}$ speed of the streak camera provided by the Hamamatsu Corporation. The data are fit to a second order polynomial which is used as the time calibration of the streak camera profiles. file is corrected for time width of each bin (pixel) and this is done by dividing the background subtracted intensity by the calibration curve. The image intensity $j$ at time $t_{i}$ is corrected by

$$
\operatorname{Bin}_{j}\left(t_{i}\right)=\frac{\operatorname{Bin}_{j}(I)-I_{0}}{\operatorname{Calib}(I)},
$$

where $I_{0}$ is the background determined from the fit to the data. The calibration curve $\operatorname{Calib}(I)$ and time $t_{i}$ are given by

$$
\begin{aligned}
\operatorname{Calib}(I)= & 1.3647-9.6146 \times 10^{-4} I \\
& +6.9609 \times 10^{-7} I^{2}, \\
t_{i}= & \int \operatorname{Calib}(I) d I \\
= & 1.36471 I-4.8073 \times 10^{-4} I^{2} \\
& +2.3203 \times 10^{-7} I^{3},
\end{aligned}
$$

for pixel $I$.

The steak camera's readout monitor had a 600 ps window to observe the bunch distribution. This $600 \mathrm{ps}$ window allowed the time calibration of the camera to be confirmed by measuring the beam location on the streak camera and varying the trigger signal. The trigger signal for the streak camera had a fine delay of $82 \mathrm{ps}$ and adjusting the trigger in $82 \mathrm{ps}$ steps, for a total of seven trigger settings, allowed the time calibration to be measured. In actuality, only six trigger settings were used because the image was not visible in the window for the seventh trigger setting.

The measurement consisted of taking 15 streak camera pictures at each trigger time, for a total of 90 pictures,

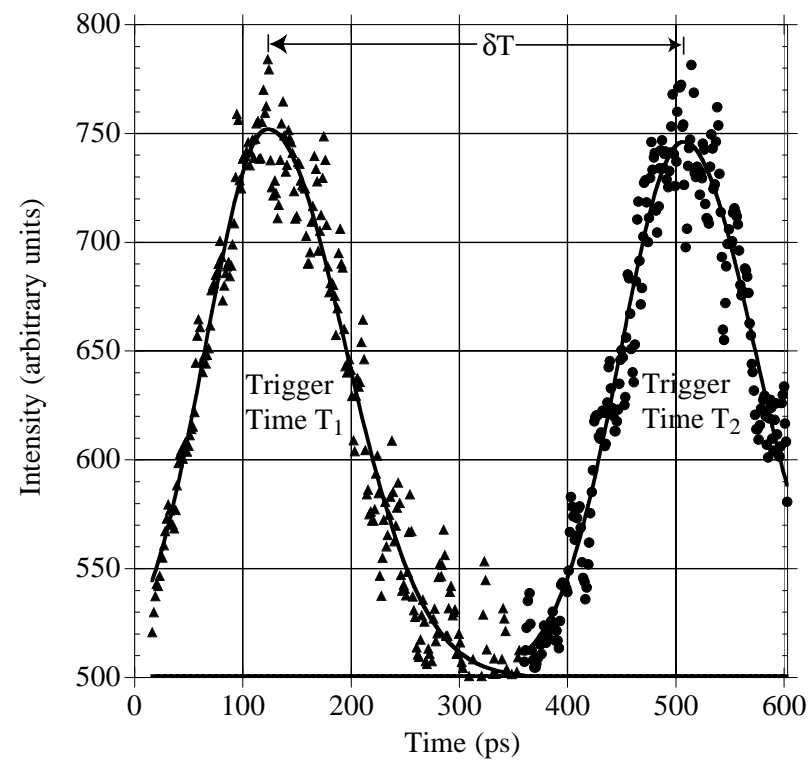

FIG 14. The sum of 15 streak camera pictures for two separate trigger settings, $T_{1}$ and $T_{2}$, where the trigger settings were separated by 410 ps. 
when the streak image was visible in the 600 ps window. The 90 pictures were taken under constant machine conditions to avoid any systematic error associated with unstable running. The 15 images from each trigger setting were fit to an asymmetric Gaussian function and the average of the 15 mean positions was calculated to determine the mean location of the bunch relative subsequent trigger settings.

The time difference on the camera was then determined from the calibration curve (Fig. 13) and compared with the trigger delay. Two of the data sets, which are separated by five $82 \mathrm{ps}$ (for a total of $410 \mathrm{ps}$ ) trigger settings, are plotted in Fig. 14. The mean difference measured by the streak camera between the two trigger settings is $\delta t=$ $T_{2}-T_{1}=419.9 \pm 11.2 \mathrm{ps}$ which is in agreement with the expected value.

\section{B. Resolution}

The streak camera resolution at zero slit width has been measured for the two fastest streak speeds $(60$ and $200 \mathrm{ps} / \mathrm{mm}$ ) at the Stanford Linear Accelerator Center (SLAC) for the Stanford linear collider using a titanium sapphire 200 fs pulsed laser [8]. The bunch length at CESR is approximately 3 times larger than the SLAC damping ring so a slower streak speed was used, $500 \mathrm{ps} / \mathrm{mm}$, and the resolution of the camera was not measured using the pulsed laser at the slower streak speed. Instead, the resolution can be determined by using the data from the faster streak speed with the calibration curve from the slower streak speed to fit the data. This is a viable alternative because the zero slit resolution is approximately $1 \%$ of the total streak time and the slit size determines the rest of the resolution component. Therefore the resolution at the $500 \mathrm{ps} / 10 \mathrm{~mm}$ speed uses the following methodology: (i) The zero slit resolution is assumed to be $1 \%$ of the full streak speed, which means $6.03 \mathrm{ps}$ [10]. (ii) The functionality of slit size can be determined by measuring the bunch length as a function of slit size for the faster streak speed laser pulses. By this methodology, the bunch length as a function of slit for the $500 \mathrm{ps} / 10 \mathrm{~mm}$ streak speed is shown in Fig. 15(a). Most of the measurements were conducted on CESR with a slit width of $200 \mathrm{~mm}$. The resolution correction is determined from Fig. 15(b).

\section{Systematic errors associated with the streak camera}

A description of the known systematic errors associated with this streak camera, such as chromatic and space charge effects, are described in detail elsewhere [8]. A systematic error associated with the camera's sensitivity was discovered while analyzing the time calibration data. A test of the streak camera's sensitivity (due to either the photocathode or multichannel plate) is to measure the sensitivity of these devices by summing up all of the
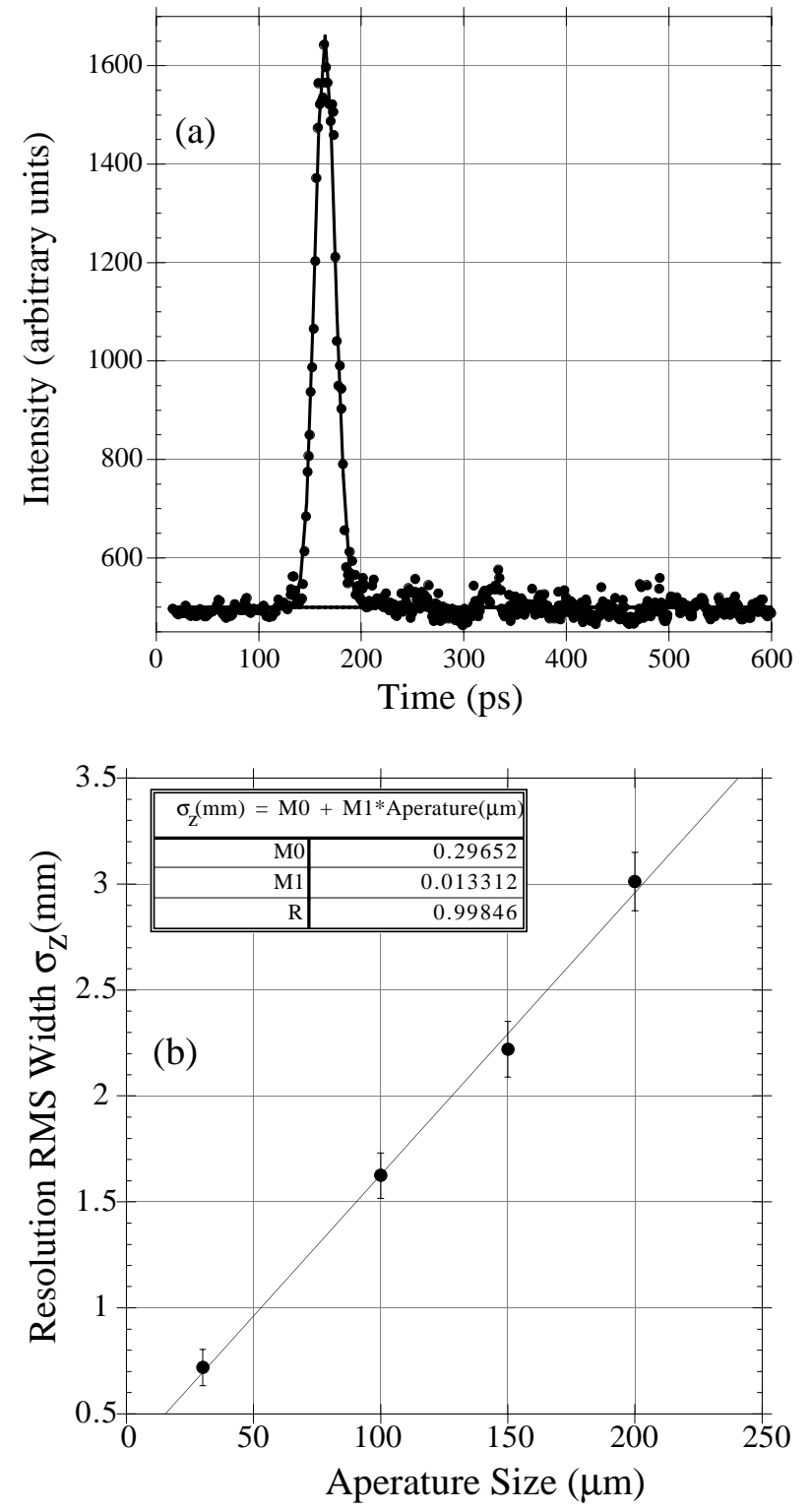

FIG. 15. (a) A single picture of the longitudinal distribution of the pulsed laser with the streak camera when the slit of the camera is set to $200 \mu \mathrm{m}$. The fit of the pulse determines the resolution of the camera at this slit size. In this particular picture the rms pulse width is $\sigma_{t}=10.06 \mathrm{ps}$. (b) The resolution of the streak camera as a function of slit size for the $500 \mathrm{ps} / 10 \mathrm{~mm}$ streak speed.

pictures taken during the time calibration measurement. Each pixel was summed in the following manner:

$$
P_{\text {total }}(i)=\sum_{j=1}^{n} P_{j}(i),
$$

where $P_{\text {total }}(i)$ is the total intensity for pixel $i$ for $n$ pictures and $P_{j}(i)$ is the intensity for pixel $i$ for a picture $j . \quad P_{\text {total }}(i)$ is plotted for all 90 pictures taken during the linearity measurement, and it is evident that pixels between number 195 and 278 have a reduced sensitivity to the input signal. Correcting pixels 195278 is done by fitting the summed image to a third 

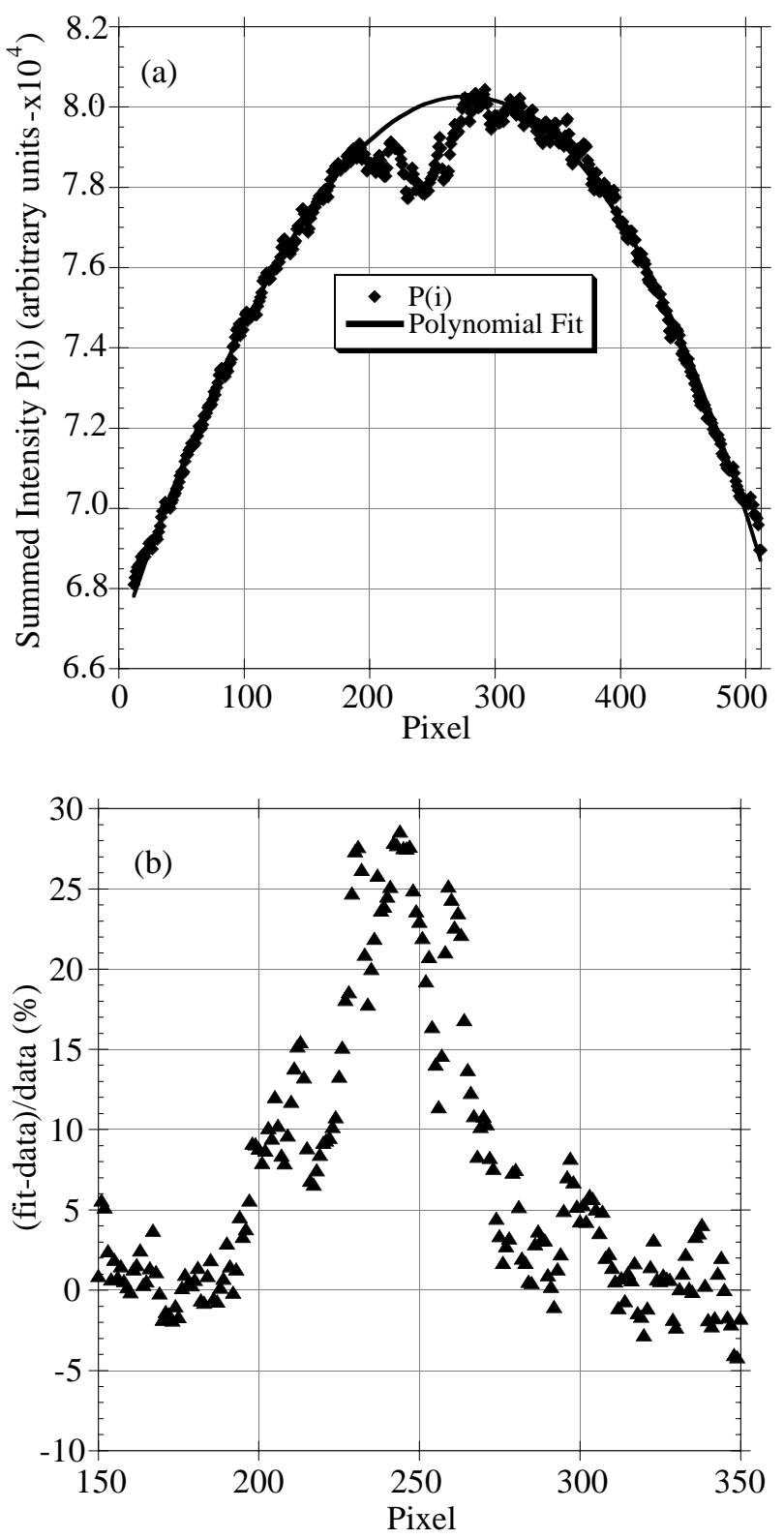

FIG. 16. (a) A pixel sum of all the streak camera pictures taken during the linearity experiment. (b) The residual between the polynomial fit and the data. The residual shown here is used to correct the sensitivity. The pixels between 195 and 278 are enhanced by the amount shown in (b).

order polynomial and adjusting each pixel by the residual shown in Fig. 16(a). Before the sensitivity correction, the bunch length and mean position on the streak camera were highly correlated. Enhancing pixels 195-278 eliminated this correlation by the amount shown in Fig. 16(b).

\section{CESR SINGLE BUNCH LONGITUDINAL DYNAMICS}

The following measurements were made on CESR with the streak camera: (A) the bunch length at low current,
(B) the bunch length as a function of current with the wiggler magnets closed and open, and (C) the bunch length as a function of $\mathrm{rf}$ accelerating voltage at low and high current.

\section{A. Low current bunch length}

The longitudinal distribution at low current is valuable due to minimized collective effects, and this gives the opportunity to compare the CESR model with the time calibration of the streak camera.

The low current bunch distribution for electrons was measured by taking approximately 15 pictures with a single bunch in CESR. Measurements were made on the electrons when the wiggler magnets were open and closed and the $\mathrm{rf}$ accelerating voltage was $V_{\mathrm{rf}}=6.87 \pm 0.01 \mathrm{MV}$. Since the measurements were made on electrons, there is a pretzel orbit change between the wiggler open and closed conditions. The light intensity was low for these measurements and this required that the streak camera slit be set to $300 \mu \mathrm{m}$ instead of $200 \mu \mathrm{m}$ (the normal setting) for greater light acceptance. The longitudinal profiles were fit to an asymmetric Gaussian function with a constant background. A single snapshot of the bunch distribution with the wiggler open and closed is shown in Figs. 17(a) and 17(b). The mean bunch length and asymmetry factor for the two cases is shown in Table VI.

The results at low current provide the following information:

(i) The mean peak of the longitudinal distribution differs between the open and closed wiggler magnet cases by $33 \%$. This difference in distribution peaks can be attributed to the acceptance of synchrotron light optics. The electron beam's pretzel orbit changes when the wiggler magnets are turned on and this changes the acceptance of the synchrotron light. The light intensity was observed to go down when the wiggler magnets were closed, and that corresponds to a lower peak in the distribution for the wiggler closed case.

(ii) A comparison of the streak camera measurements to the CESR model is made in Table VI. Ignoring collective effects of the beam, the theoretical bunch length is $3.3 \%$ smaller than the measured bunch length when the wiggler magnets are closed and $2.1 \%$ smaller than the measured bunch length when the wiggler magnets are open. This difference between the measured bunch length and the CESR model is possibly due to an incorrect calibration of the sweep voltage for the streak camera or a systematic shift in the rf voltage calibration. A 5\% error in the rf voltage calibration would resolve the difference between the measured bunch length and the CESR model bunch length.

(iii) The residuals from the fit can be used to determine if there is any bunch distribution motion. Figures 18(a) and 18(b) are pictures of the difference between a single picture of the bunch distribution and the average 
TABLE VI. The CESR low current bunch length results with wiggler magnets open and closed.

\begin{tabular}{llrcc}
\hline \hline & rms $\sigma_{z}(\mathrm{~mm})$ & Asymmetry factor & Distribution peak & CESR model $\sigma_{z}(\mathrm{~mm})$ \\
\hline Wigglers closed & $17.89 \pm 0.35$ & $-0.020 \pm 0.022$ & $70.8 \pm 2.7$ & 17.39 \\
Wigglers open & $15.91 \pm 0.12$ & $-0.0024 \pm 0.029$ & $106.7 \pm 3.6$ & 15.65 \\
\hline \hline
\end{tabular}

bunch distribution when the wiggler magnets are open and closed.

The sum of all the residuals is shown in Figs. 19(a) and $19 \mathrm{c}(\mathrm{b})$. It is interesting to note that the background curvature is more evident in the case when the wiggler magnets were open, and this could be due to high intensity
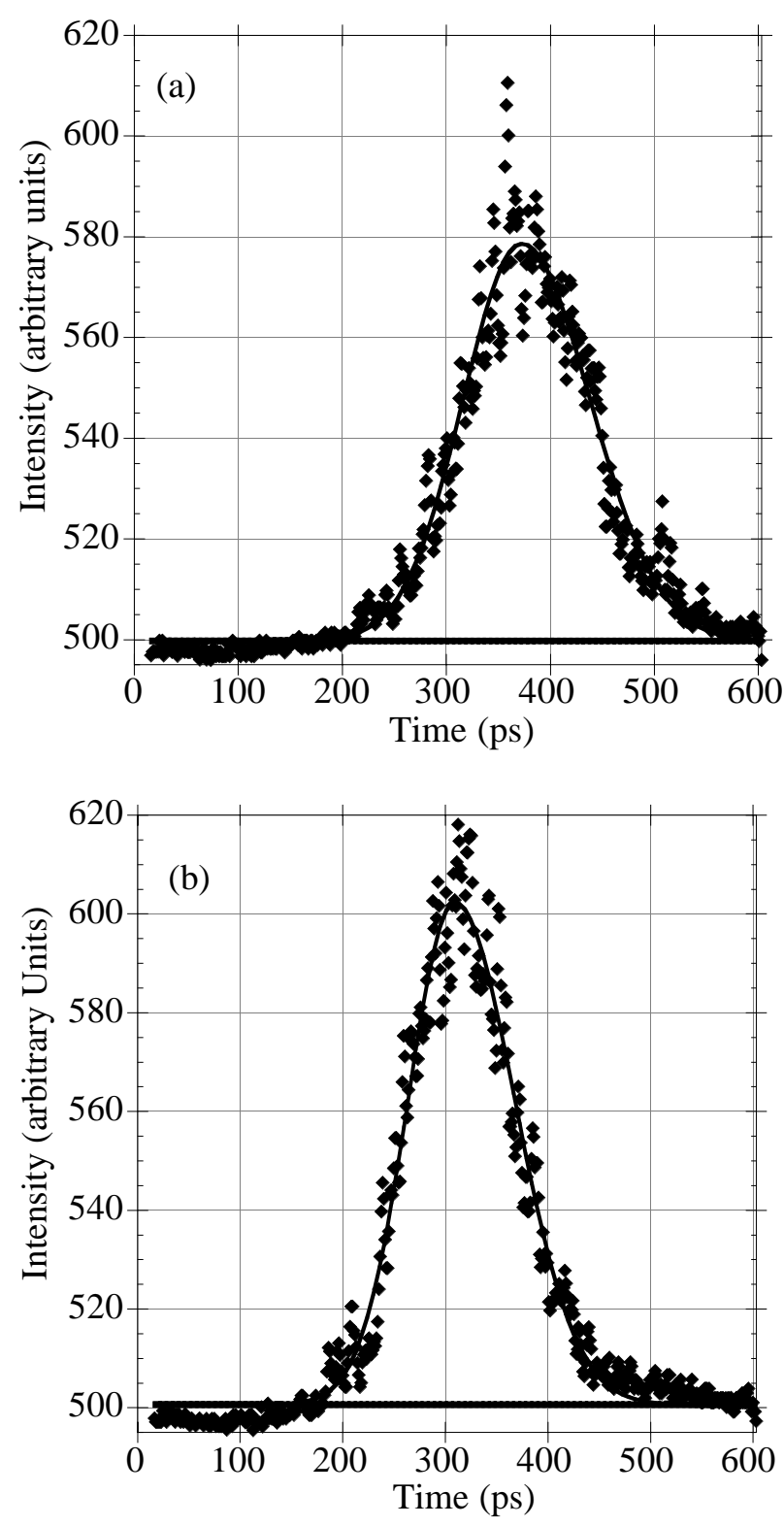

FIG. 17. A single picture of the bunch distribution for CESR with (a) the wiggler magnets closed, a current of $1.4 \mathrm{~mA}$, and rf accelerating voltage at $6.87 \mathrm{MV}$, and (b) the wiggler magnets open, a current $1.4 \mathrm{~mA}$, and rf accelerating voltage of $6.87 \mathrm{MV}$. levels. The deviation from the average distribution is not greater than $9 \%$, and this can be attributed to the fluctuations in the light and noise in the camera. This deviation can be compared with higher current data. It should also be noted that no instability has been observed with the residual data.
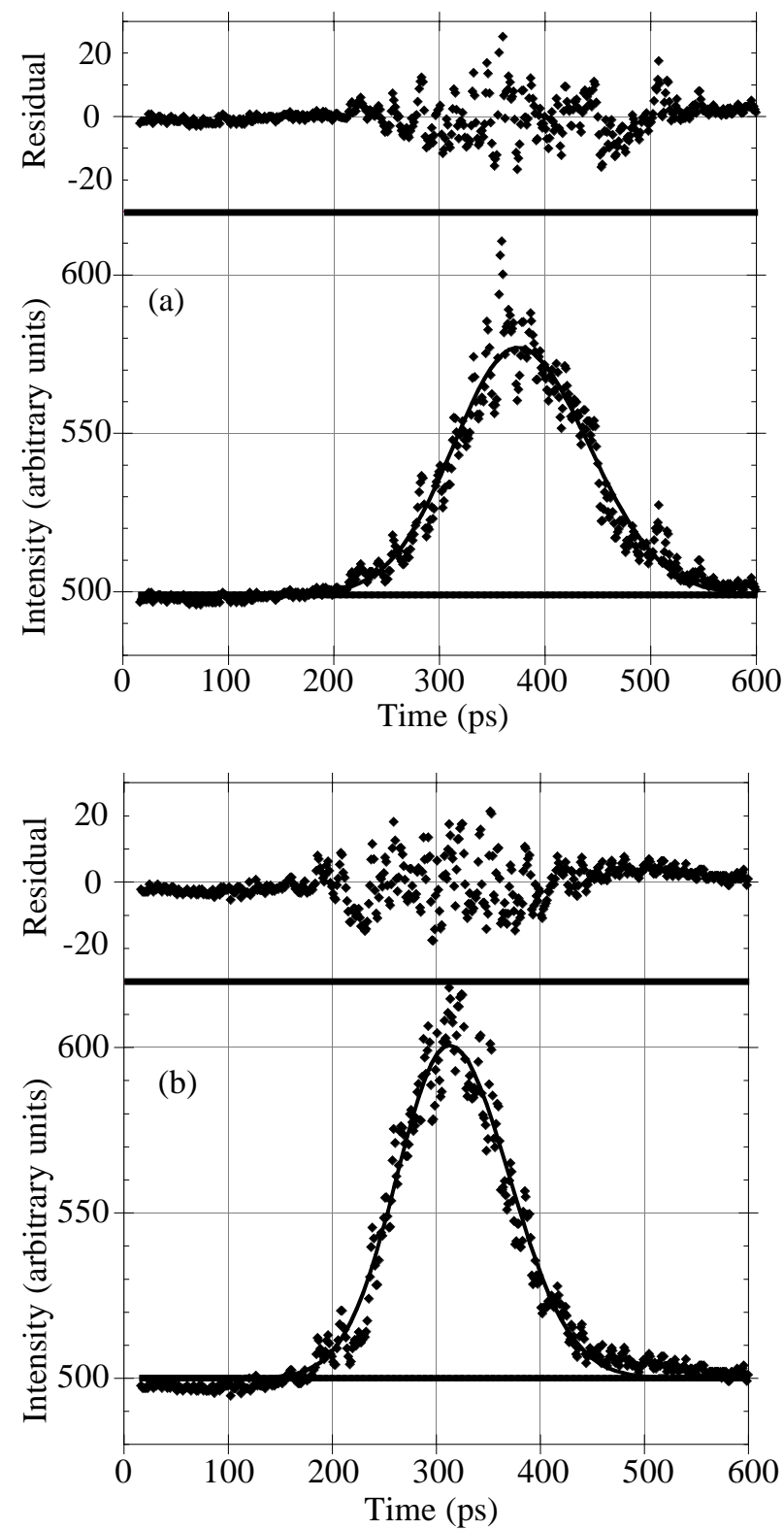

FIG. 18. Single streak camera pictures fit to the mean with the width and asymmetry held constant. The residual from the fit is plotted above the distribution for the case when the wiggler magnets are (a) closed and (b) open. 

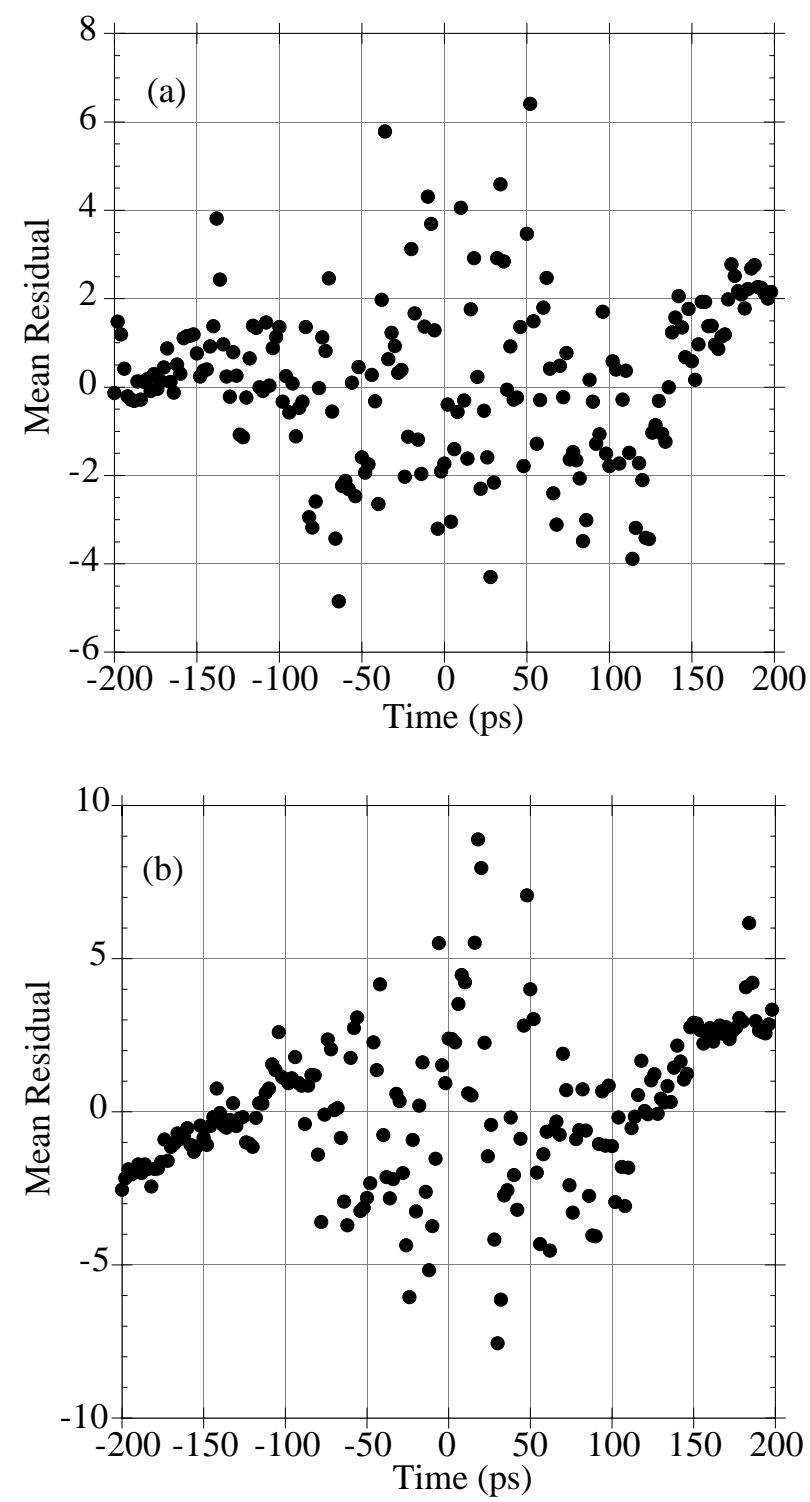

FIG. 19. The mean residual for the streak camera data set at low current when the wiggler magnets were (a) closed and (b) open. The current and rf voltage were the same as stated in Fig. 17.

\section{B. Bunch length as a function of current}

The electron bunch distribution was measured as a function of current with both wigglers open and closed. These measurements consisted of taking approximately 15 streak camera pictures at each current setting between the currents of $1 \mathrm{~mA}$ up to $35 \mathrm{~mA}$ with a single bunch in CESR and an rf setting of $V_{\mathrm{rf}}=6.87 \pm 0.01 \mathrm{MV}$. The light intensity increases as a function of current so neutral density filters were used to avoid saturation of the camera. The slit width of the streak camera was set to $200 \mu \mathrm{m}$, except at the lowest current setting when it was $300 \mu \mathrm{m}$. The profiles were fit to an asymmetric Gaussian function with a constant background and mean bunch length and asymmetry were computed. The mean length and asymmetry were used to compute the average residual from the set of data. A plot of the bunch length and asymmetry factor as a function of current with the wigglers open and closed is shown in Figs. 20(a) and 20(b).

There are several noteworthy features of the experimental data.

(i) The bunch length increases linearly with current, denoted by the fit to the data in Fig. 20(a), when the wigglers are open, but when the wigglers are closed the bunch length remains fairly constant until approximately $10 \mathrm{~mA}$, when the length increases dramatically. There is a $12 \%$ growth in the bunch length between the currents of $1-30 \mathrm{~mA}$. This bunch lengthening is due to potential well distortion, and the CESR vacuum chamber impedance can be determined from this data.
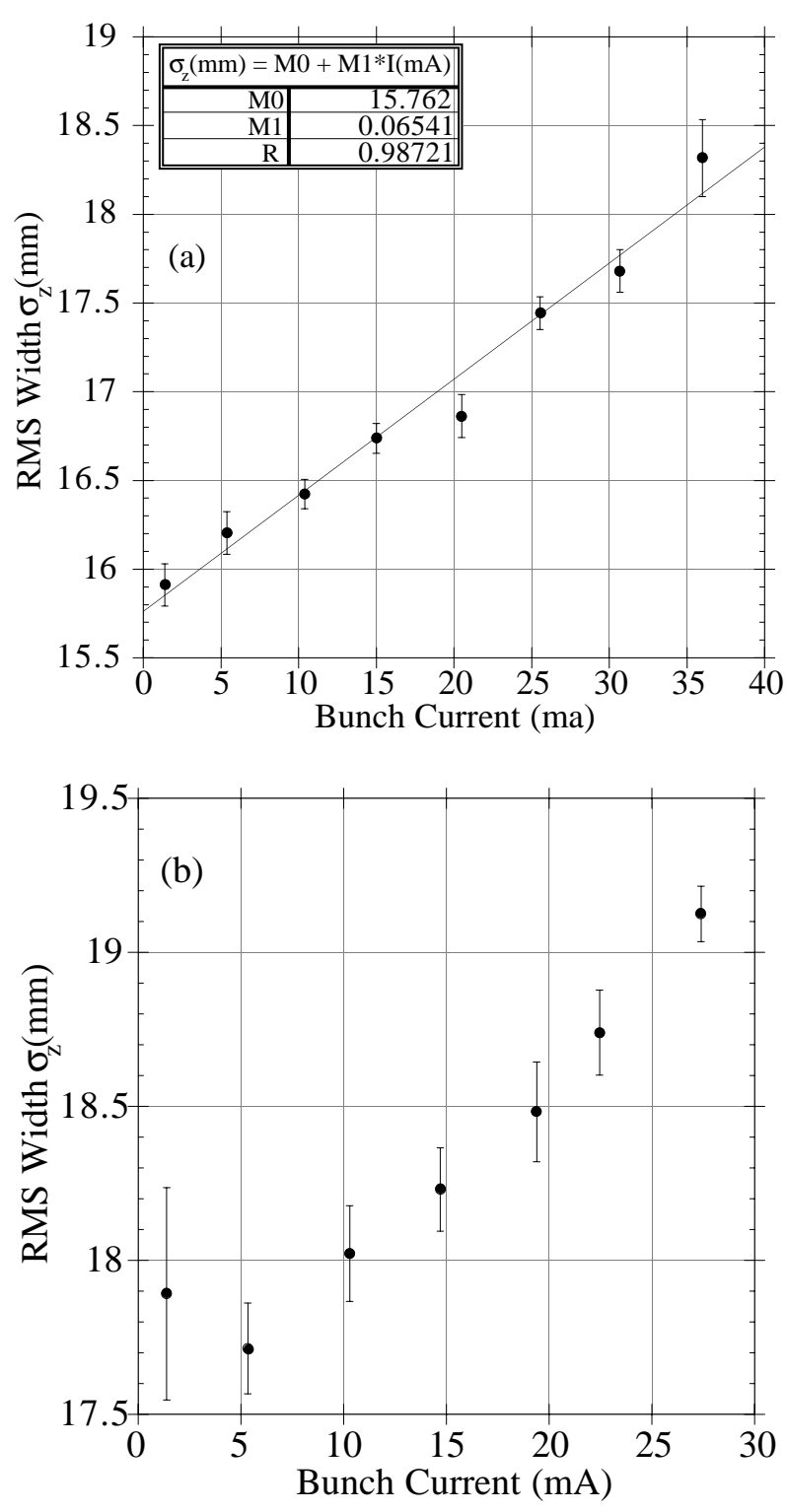

FIG. 20. The bunch length in CESR as a function of current when the wiggler magnets are (a) open and (b) closed. 
(ii) The asymmetry factor [shown in Figs. 21(a) and 21(b)], which measures the departure from a Gaussian distribution increases in both cases as a function of current. The asymmetry factor when the wigglers are open or closed is linear with current. The growth rate for the asymmetry factor in both cases is approximately the same.

(iii) The current threshold for this experiment was not due to an instability but was a result of an increase in the CLEO detector background and a poor beam lifetime, which made it unimaginable to store the beam. To observe an instability during these measurements, two methods of detection were used. (a) The spectrum analyzer is the normal method of detecting a longitudinal
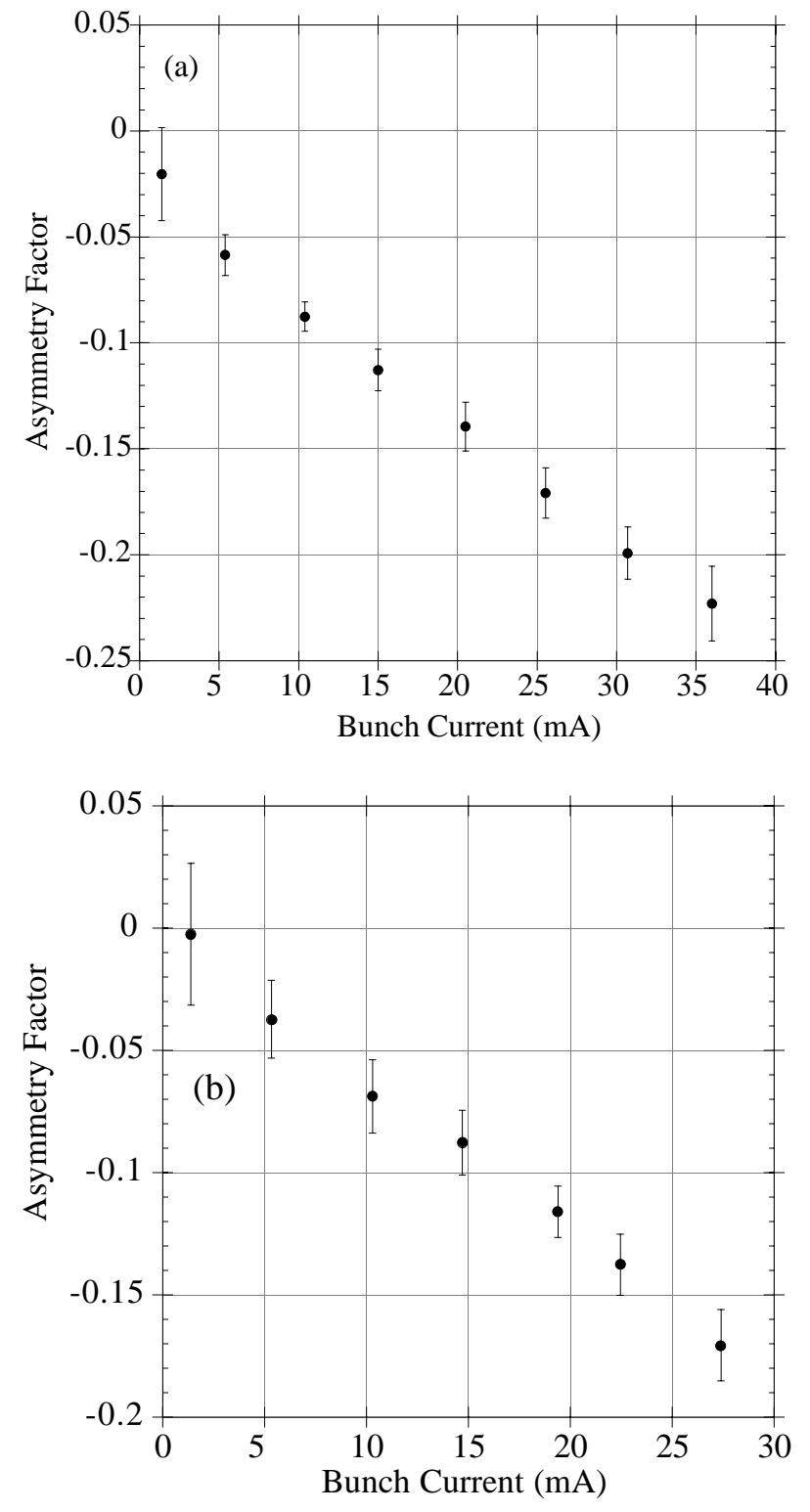

FIG. 21. The asymmetry factor as a function of current in CESR when the wiggler magnets are (a) open and (b) closed.
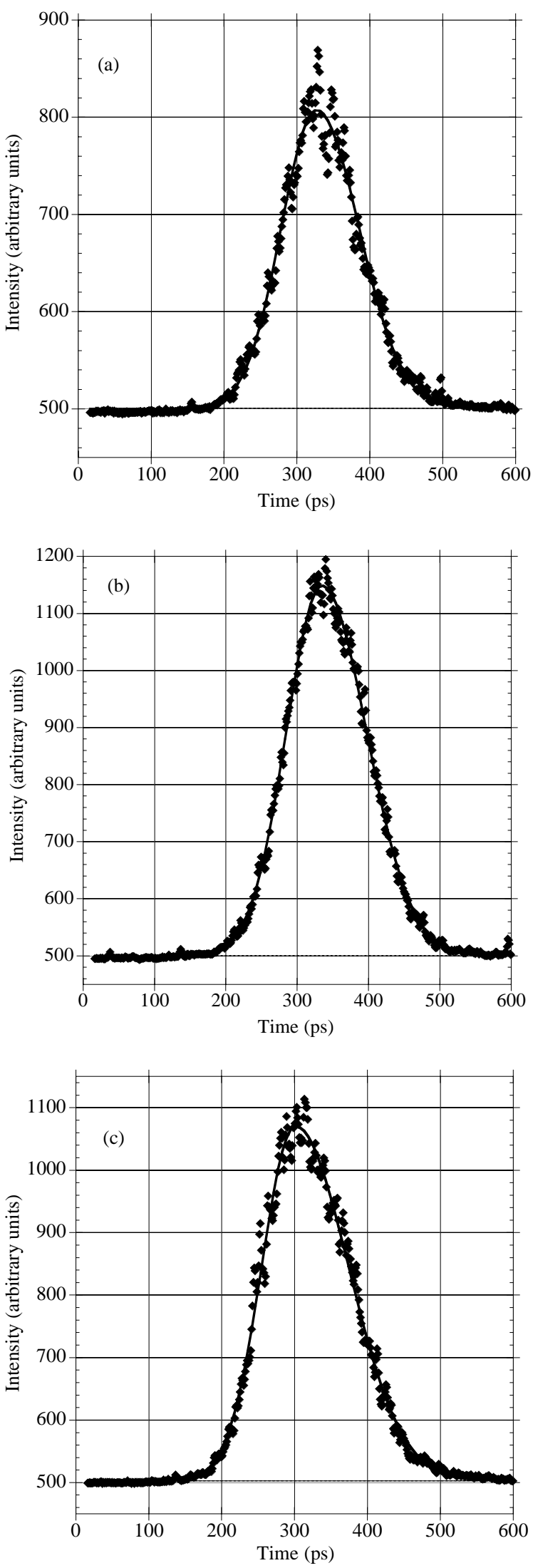

FIG. 22. A single streak camera picture of the CESR bunch distribution when the wiggler magnets are open and the single bunch current is (a) $5.4 \mathrm{~mA}$, (b) $15.0 \mathrm{~mA}$, and (c) $30.5 \mathrm{~mA}$. 

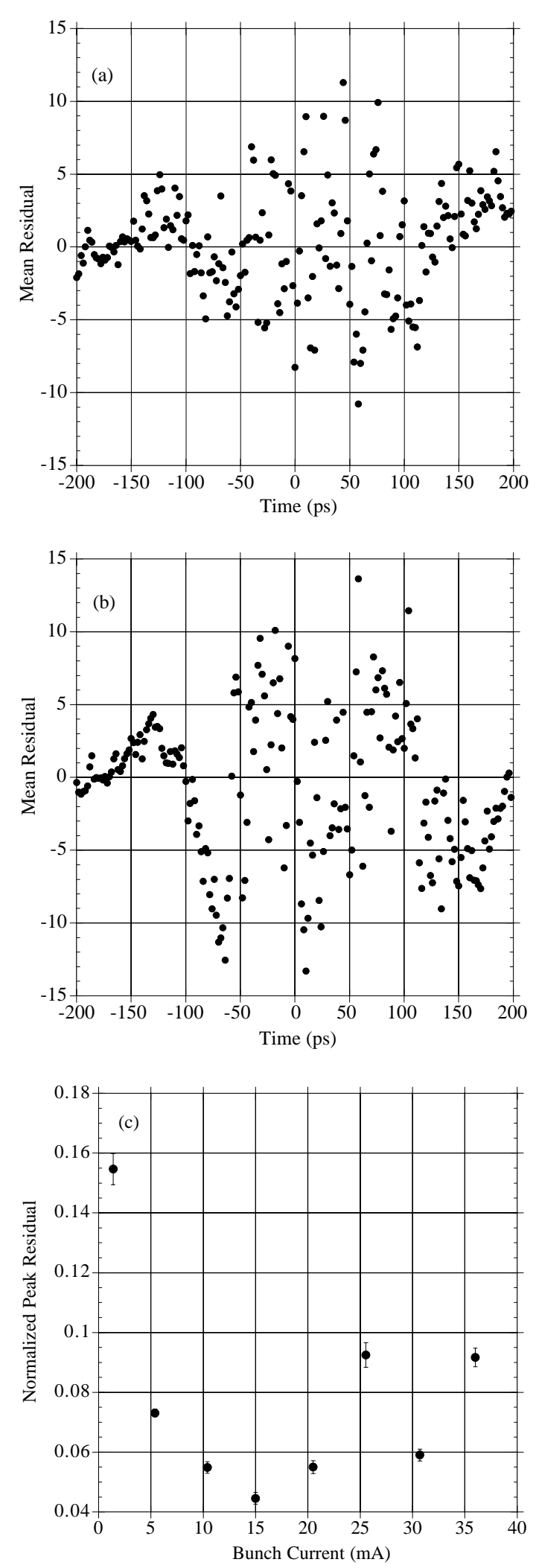

FIG. 23. The mean residual from the fit to the data for a single bunch current of (a) $5.4 \mathrm{~mA}$ and (b) $30.7 \mathrm{~mA}$ and wiggler magnets open. (c) The peak mean residual normalized by the peak distribution as a function of current for single bunch and wiggler magnets open. The error bars are determined from the spread in the peak of the distribution. instability by observing sidebands off of the revolution harmonics. During these experiments no sidebands were observed. (b) The second method of instability observation is to look at the individual distributions and residuals measured by the streak camera. Figures 22(a)-22(c) are single pictures, at three different currents, fit to an asymmetric Gaussian function.

The average or mean residuals from the fit to the data are also used to determine if an instability was present. Figures 23(a) and 23(b) are plots of mean residuals for low current and high current when the wiggler magnets were open. The amplitude of the residual increased slightly with current, but the light intensity also increased. If the amplitude (peak to peak) of the residual is normalized (divided) by the peak of the distribution there is a decrease in the residual as a function of current [Fig. 23(c)]. It appears that as the current increases there is more structure in the residual, but it also could be due to the increase in intensity.

iv) The tail of the distribution gets longer as the current increases, which is a signature of potential well distortion due to the resistive impedance of the vacuum chamber. These distributions can be used to extract the vacuum chamber impedance of CESR.

\section{Bunch length as a function of $\mathrm{rf}$ accelerating voltage}

At low current, collective behavior of the bunch is small, and as a result the equilibrium bunch length in a storage ring is approximately inversely proportional to the square root of the $\mathrm{rf}$ accelerating gap voltage. At low current, the electron bunch length in CESR was measured as a function of $\mathrm{rf}$ accelerating voltage. This was done by taking ten streak camera pictures with a streak camera slit of $200 \mu \mathrm{m}$ at each rf voltage between 4.7 and 6.5 MV. The mean and root mean error were calculated at each rf setting and plotted in Fig. 24(a). Fitting the data to the function $\sigma_{z}=A\left(V_{\mathrm{rf}}\right)^{m}$ gives a value of $m=-0.43 \pm 0.02$.

The electron bunch length versus rf accelerating voltage at high current was measured with the streak camera by taking 12 streak camera profiles with a camera slit set at $200 \mu \mathrm{m}$ at each rf voltage and the profiles were fit to an asymmetric Gaussian function. The mean and root mean error were calculated at each rf setting and plotted in Fig. 24(b). Fitting the data to the function $\sigma_{z}=A\left(V_{\mathrm{rf}}\right)^{m}$ gives a value of $m=-0.40 \pm 0.04$.

From comparing the low and high current results it can be concluded that the dependence of the bunch length on the rf accelerating voltage does not change appreciably as the current changes in CESR. 

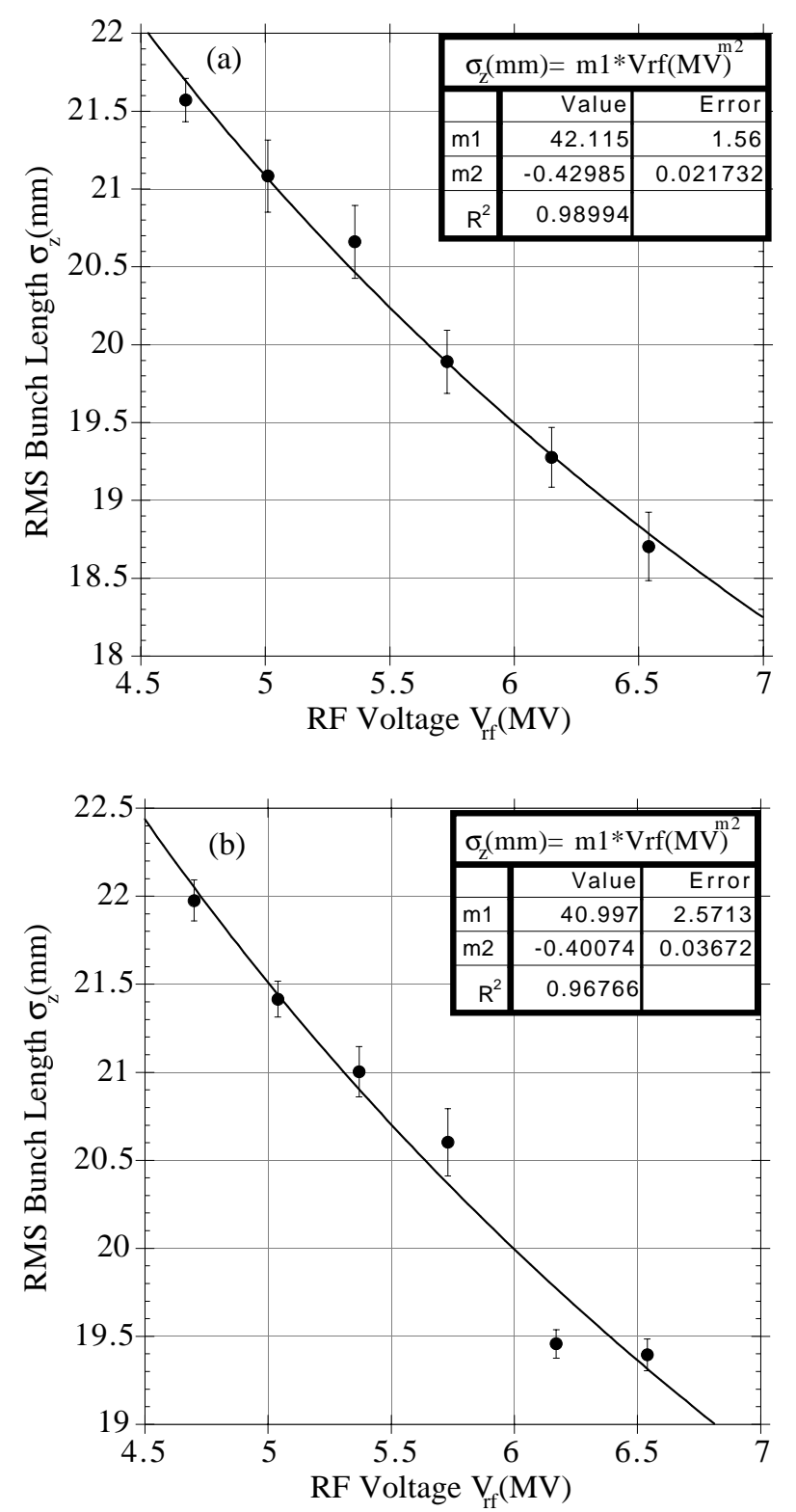

FIG. 24. The bunch length as a function of $\mathrm{rf}$ accelerating voltage when the current in CESR is (a) $3.2 \mathrm{~mA}$ and (b) $15.0 \mathrm{~mA}$.

\section{DATA ANALYSIS - INFERRING THE CESR VACUUM CHAMBER IMPEDANCE}

\section{A. Bunch length as a function of current}

Under the assumption that the resistance and inductance are constant over the measured range of the bunch lengths, the resistive and inductive impedance components of the CESR storage ring vacuum chamber are determined from a $\chi^{2}$ fit between the measured bunch distributions and the simulated bunch distributions. The $\chi^{2}$ fit is given by

$$
\chi^{2}=\sum_{i=1}^{n} \frac{\left(S\left(t_{i}\right)-M\left(t_{i}\right)\right)^{2}}{S\left(t_{i}\right)}
$$

where $S\left(t_{i}\right)$ and $M\left(t_{i}\right)$ are the simulated and measured bunch height at time $t_{i}$ in the distribution. From the $\chi^{2}$ fit, the resistance and inductance for each current setting is inferred. An example of the $\chi^{2}$ fit is shown in Figs. 25(a) and $25(\mathrm{~b})$.

From the $\chi^{2}$ fit to the data, the resistance for the CESR ring is

$$
\begin{gathered}
R_{\text {open }}=1523 \pm 343 \Omega, \\
R_{\text {closed }}=1322 \pm 310 \Omega,
\end{gathered}
$$
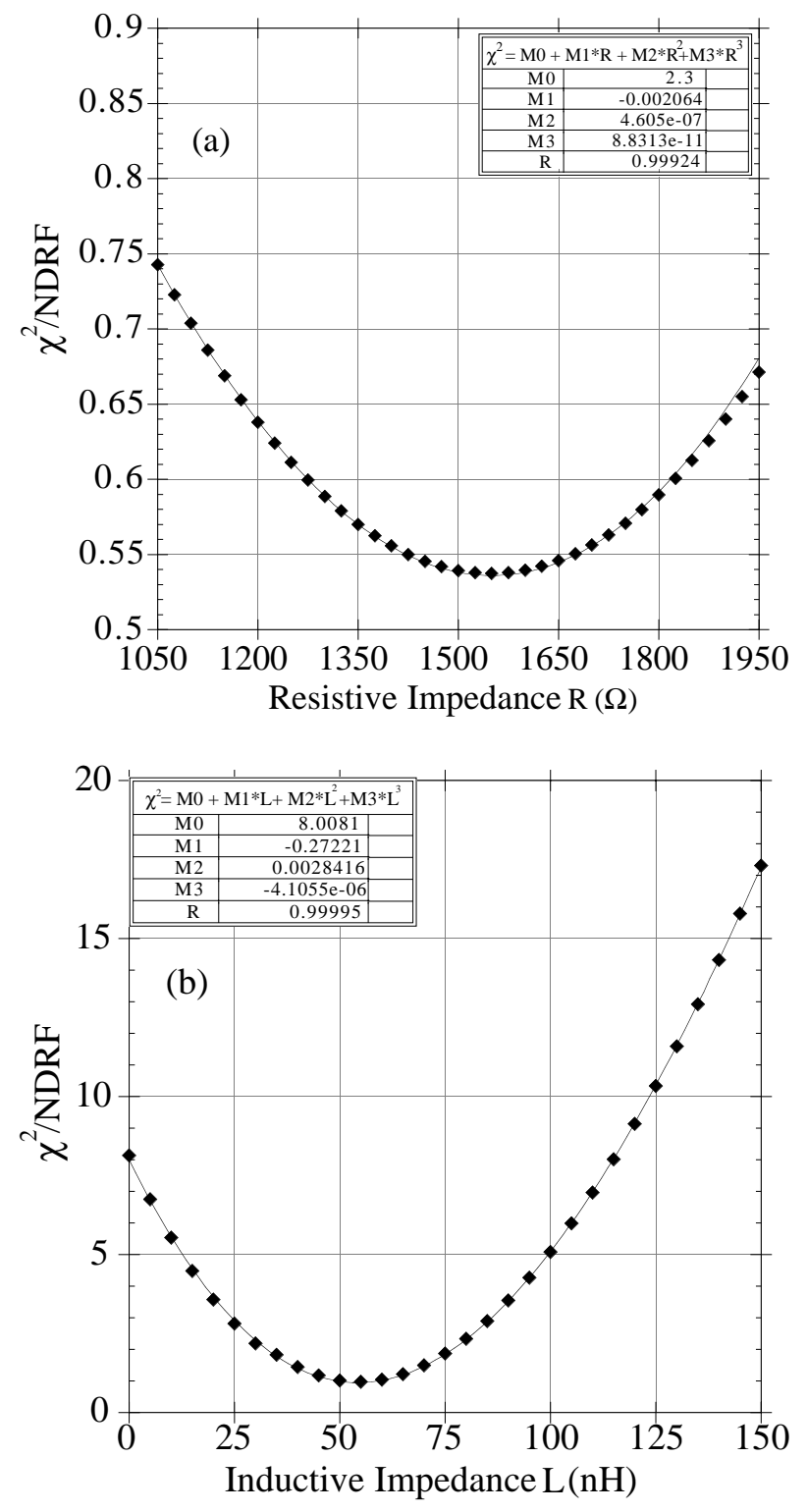

FIG. 25. The results from the $\chi^{2}$ fit for (a) a current of $15 \mathrm{~mA}$ and inductance of $65 \mathrm{nH}$ and (b) a single bunch current of $15 \mathrm{~mA}$ and resistance of $1537 \Omega$. The resistance and inductance are calculated from fitting the data to a third order polynomial and finding its minimum. In this instance, the resistance in plot (a) is $1550 \Omega$ and the inductance in plot (b) is $54 \mathrm{nH}$. 
and the inductance is

$$
\begin{aligned}
L_{\text {open }} & =65 \pm 12 \mathrm{nH}, \\
L_{\text {closed }} & =72 \pm 13 \mathrm{nH} .
\end{aligned}
$$

The uncertainties are determined by varying the simulation over the range of measurement errors on the asymmetry factor and rms width. There are several noteworthy features of the determined impedance. (i) The resistive impedance is in agreement with the higher order mode measurement [7], and (ii) there is a $13 \%$ difference between the wigglers open and closed resistive impedance.

Comparisons between the simulated bunch distributions using the above resistance and inductance and the streak camera measurements have been made. Figures 26 and 27 are the measured and simulated bunch length as a function
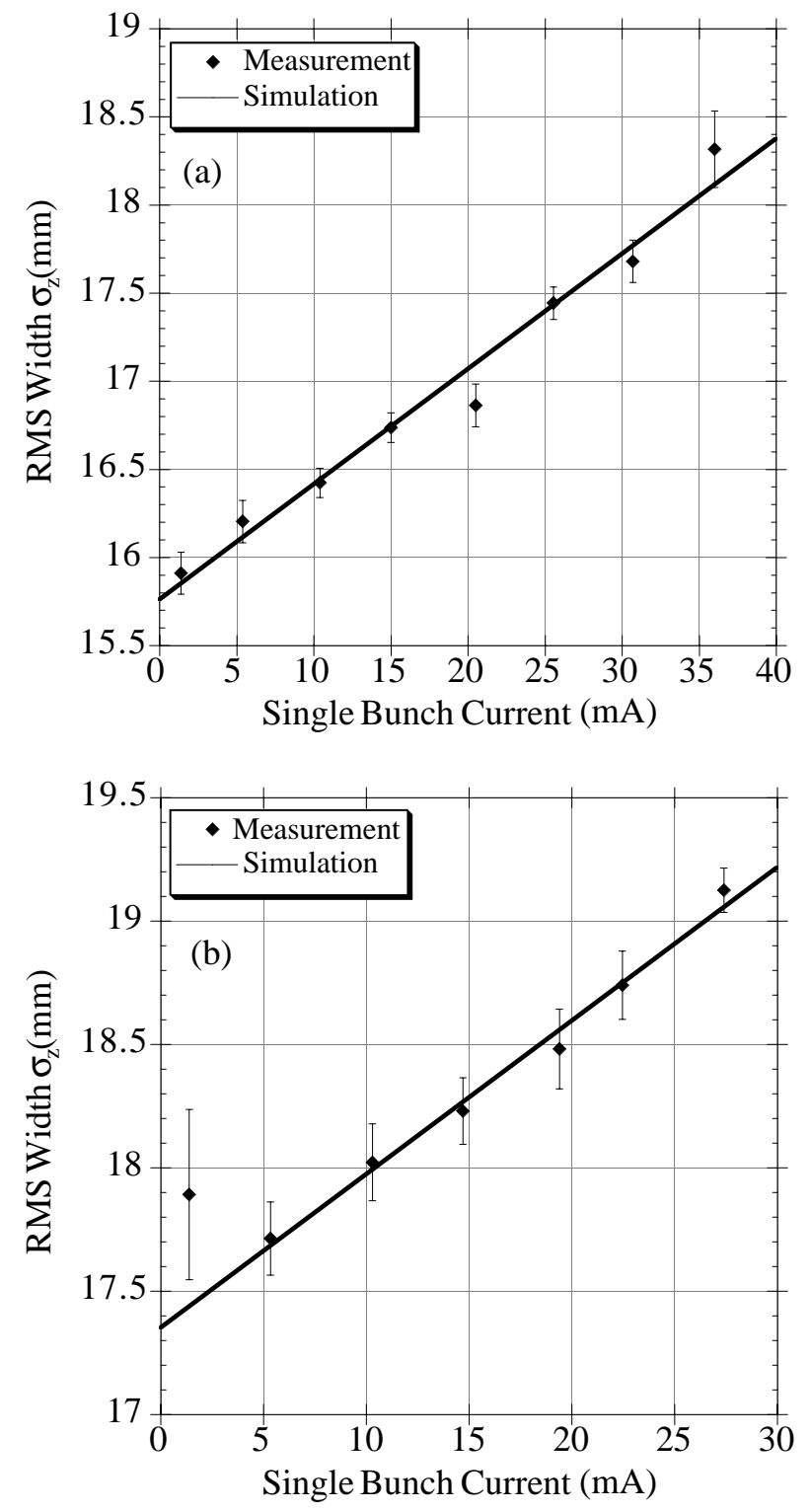

FIG. 26. The measured and simulated bunch length as a function of current when the wiggler magnets are (a) open and (b) closed.
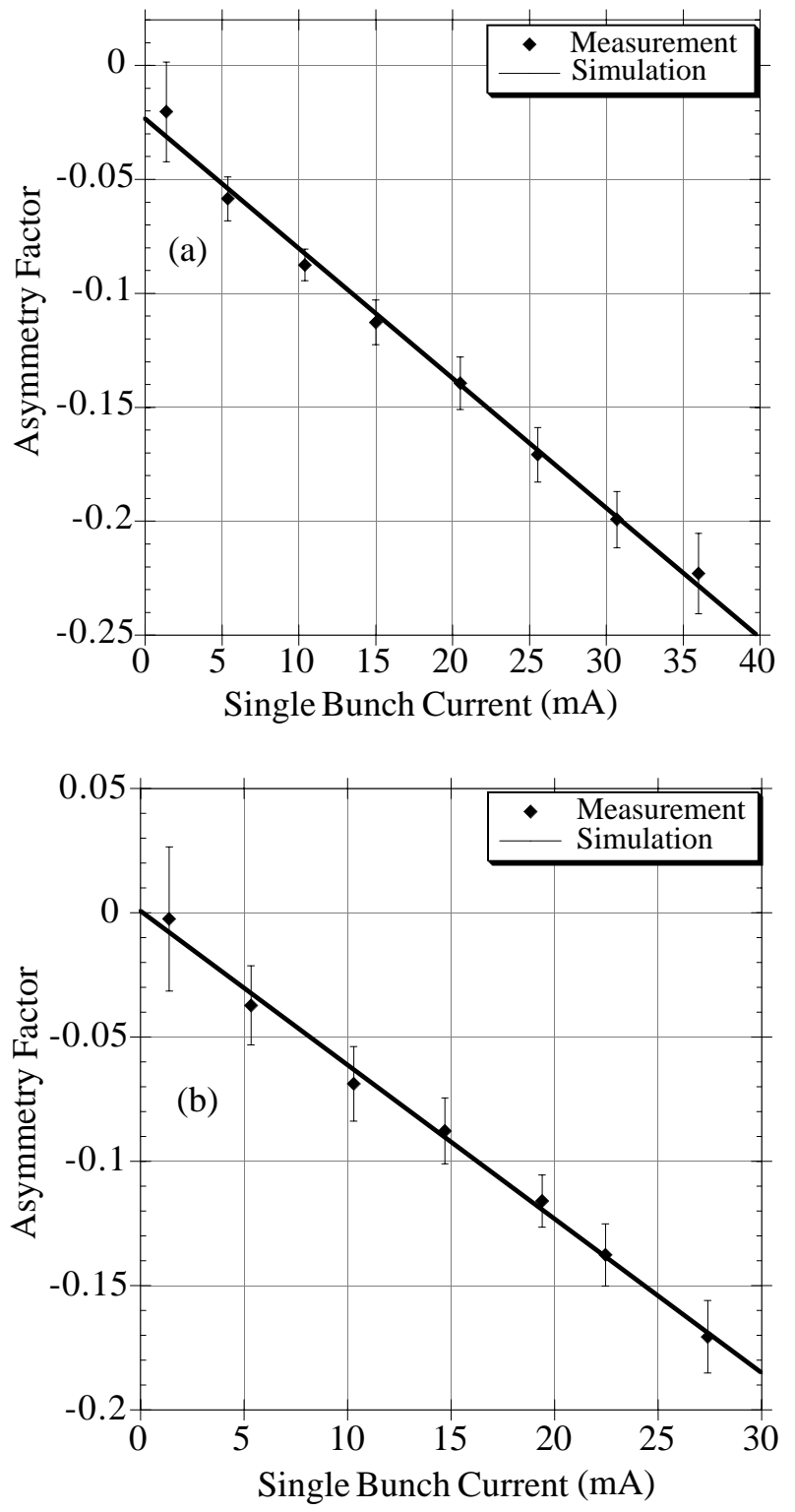

FIG. 27. The measured and simulated bunch asymmetry as a function of current when the wiggler magnets are (a) open and (b) closed.

of current, and Fig. 28 is the measured and simulated bunch length as a function of rf accelerating voltage.

\section{B. Bunch length as a function of rf accelerating voltage}

The same $\chi^{2}$ fit between the measured and simulated bunch distributions can be employed to determine the CESR vacuum chamber impedance from the data taken when the rf voltage was varied. From the $\chi^{2}$ fit to the data, the resistance for the CESR ring is

$$
R_{\text {closed }}=1468 \pm 304 \Omega,
$$

and the inductance is

$$
L_{\text {closed }}=62 \pm 14 \mathrm{nH} .
$$


Figure 28 is the measured and simulated bunch length as a function of $\mathrm{rf}$ accelerating voltage.

Comparing the simulated and measured data several comments can be made. (i) The impedance for the two measurements, bunch length versus current and $\mathrm{rf}$ accelerating voltage, agree. (ii) The upper and lower limits due to the range of impedance values are shown in Fig. 28. As expected, the limits are tighter in the low current case than in the high current case [Fig. 28(a)]. (iii) The simulated bunch length (Fig. 28) as a function of rf voltage data is fit to the function $\sigma_{z}=A\left(V_{\mathrm{rf}}\right)^{m}$. The change in the power $m$ between simulation $\left(m_{s}\right)$ and measurement $\left(m_{m}\right)$ can be determined. In the low current case $\Delta m=m_{m}-m_{s}=0$, and in the high current case $\Delta m=0.02$. The change in slope between the low and high current measurements suggests that the impedance changes the slope of the curve. The low current behavior of the bunch length is not precisely inversely proportional to the square root of the $\mathrm{rf}$ accelerating voltage; this
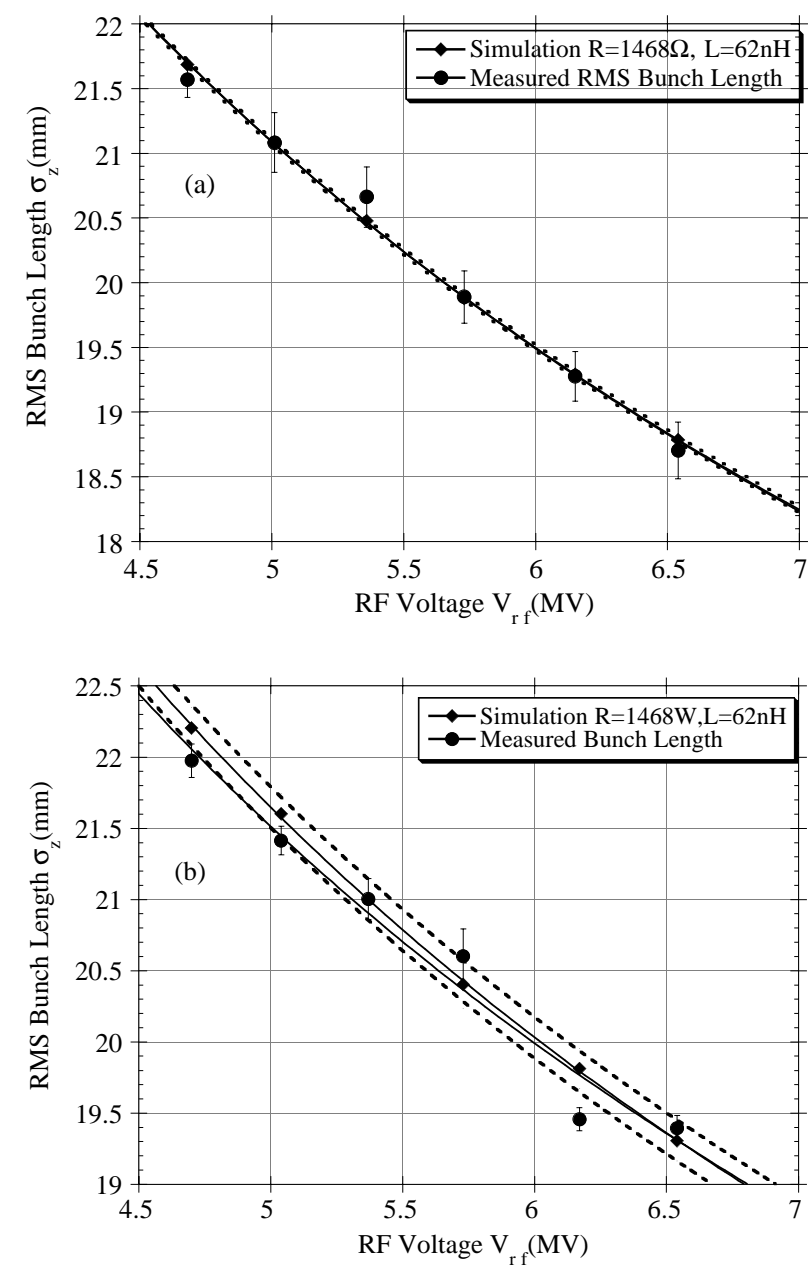

FIG. 28. The measured and simulated bunch length as a function of $\mathrm{rf}$ accelerating voltage when the current in CESR is (a) $3.2 \mathrm{~mA}$ and (b) $15.0 \mathrm{~mA}$. The dashed lines are the simulated bunch lengths for the upper and lower limits on the measured impedance. is due to the effect of the bunch changing synchronous phase and, hence, the slope of the rf voltage due to nonzero synchrotron radiation loss. At high current, the bunch length dependence on $\mathrm{rf}$ voltage deviates from the low current case slightly due to collective effects. (iv) Determining the impedance from the bunch length dependence on current, in Sec. VIII A, and the bunch length dependence on rf voltage in this section, produces results that are in good agreement.

Two examples of the simulated bunch distribution fit to an asymmetric Gaussian function are plotted in Figs. 29(a) and 29(b).
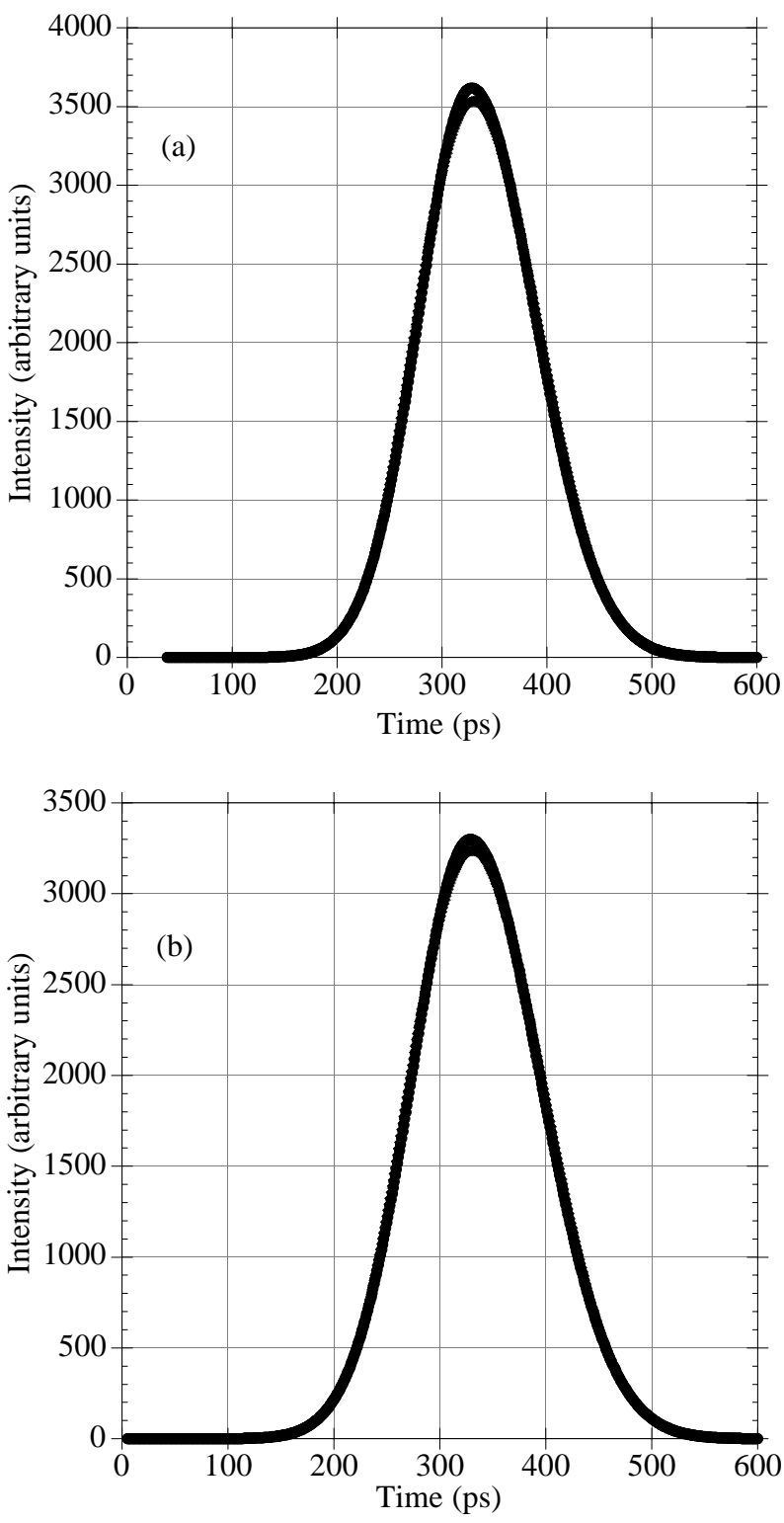

FIG. 29. The simulated CESR bunch distribution fit to an asymmetric Gaussian function with (a) the wiggler magnets open, a bunch current of $15 \mathrm{~mA}$, and impedance of $R=$ $1523 \Omega$ and $L=65 \mathrm{nH}$ and (b) the wiggler magnets closed, a bunch current of $15 \mathrm{~mA}$, and impedance of $R=1322 \Omega$ and $L=72 \mathrm{nH}$. 


\section{CONCLUSIONS}

In this paper, we have reported the results from the single bunch streak camera measurements that were done for the first time at CESR. These experiments allowed us to resolve the details of the beam distribution not available by other techniques. Furthermore, from the data obtained we were able to extract the parameters of the CESR vacuum chamber impedance, modeling it as a sum of the inductive and resistive parts. We have also established that the potential well distortion is the main single bunch collective effect in CESR. It leads to some asymmetry and lengthening of the beam distribution at high current. We have looked for single bunch coherent instabilities but have not registered any up to the highest value of current allowed by the CLEO detector background thresholds. Finally, we have also established that the wiggler magnet changes the synchrotron integrals and impedance as expected, which results in a change in bunch length.

The systematic errors associated with the streak camera are well understood, and the low current single beam bunch length measurements are in close agreement with the theoretical model of CESR. There were no observed differences between the electron and positron bunch length. The bunch length growth with current, when the wiggler magnets were closed and opened, was used to determine the impedance of CESR. The assumption that the resistive and inductive impedance is constant over the bunch lengths measured is consistent with the results. The impedance was determined by comparing the measured bunch length dependence on the current and comparing it to simulations. From this method, the vacuum chamber impedance has a resistance of $1523 \pm 343 \Omega$ and $1322 \pm 310 \Omega$ (which is in agreement with previous measurements) and an inductance of $65 \pm 12 \mathrm{nH}$ and $72 \pm 13 \mathrm{nH}$ for the wiggler magnets open and closed cases, respectively. The impedance from the bunch length dependence on current and the bunch length dependence on $\mathrm{rf}$ voltage produce results that are in good agreement. Knowing the vacuum chamber impedance allows bunch distributions to be simulated for future possible operating points.
These measurements confirm our understanding of the theoretical model of the CESR and were valuable in exploring the possible future use of streak cameras as a diagnostic tool in the CESR accelerator complex.

\section{ACKNOWLEDGMENTS}

The authors would like to thank the Stanford Linear Accelerator Center for the loan of the streak camera, especially Robert Siemann and Marc Ross. We would also like to thank the CESR technicians for their help getting the experimental area ready for the camera, the machine shop for making the copper mirror, and the vacuum group, especially Yulin $\mathrm{Li}$, for designing and installing the mirror. The work of M. S. was supported by the National Science Foundation. The work of B. P. was supported by the Department of Energy under Contract No. DE-AC03-76SF00515.

[1] E. B. Blum, R. H. Siemann, D. H. Auston, R. R. Freeman, P. Smith, and D. H. Mills, Nucl. Instrum. Methods Phys. Res. 207, 321 (1983).

[2] Z. Greenwald et al., Cornell University Report No. CON 90-22, 1990

[3] M. Sands, SLAC Report No. SLAC-PUB-0121, 1970.

[4] J. Haissinski, Nuovo Cimento Soc. Ital. Fis. 18B, 72 (1973).

[5] M. Billing, Cornell University Report No. CBN 80-2, 1980. The updated software was written by M. Stedinger and M. Billing, 1998.

[6] A.W. Chao, Physics of Collective Beam Instabilities in High-Energy Accelerators (Wiley, New York, 1993), p. 371.

[7] M. Billing and D. Rice (unpublished).

[8] R. L. Holtzapple, SLAC Report No. SLAC-Report-487, 1996.

[9] F. James and M. Roos, Comput. Phys. Commun. 10, $343-$ 367 (1975).

[10] B. Cieslik, Hamamatsu Photonics (private communication). 